\title{
Revealing the Conducting Character of the $\beta$-NiOOH Catalyst through Defect Chemistry
}

\author{
Kapil Dhaka ${ }^{1}$ and Maytal Caspary Toroker ${ }^{1,2^{*}}$ \\ ${ }^{1}$ Department of Materials Science and Engineering, Technion - Israel Institute of Technology, \\ Haifa, 3200003, Israel \\ ${ }^{2}$ The Nancy and Stephen Grand Technion Energy Program, Technion - Israel Institute of \\ Technology, Haifa 3200003, Israel
}

\section{Supporting Information}

\section{Elemental Energies:}

Several corrections are added to the total energy of the elemental atom's energies of $\mathrm{Ni}, \mathrm{O}$, and $\mathrm{H}$. In case of Nickel atom energy- Nickel is generally found in Ni-fcc structure. To find the ground state energy, following thermal, vibrational and entropy corrections are introduced:

$$
\mu^{\mathrm{ele}}(N i)=\frac{1}{4}\left[E_{N i(f c c)}+\frac{1}{2} \sum_{N=1}^{3(N-1)-3} h v_{i}+\sum_{N=1}^{3(N-1)-3} \frac{h v_{i}}{e^{\frac{h v_{i}}{k T}}-1}-T \Delta S_{N i(f c c)}\right]
$$

Where, the $1^{\text {st }}$ term is the total energy of the $\mathrm{Ni}$-fcc unit cell which has $4 \mathrm{Ni}$ atoms, $2^{\text {nd }}$ term denotes the zero-point energy (ZPE) corrections for the vibrational frequencies, $3^{\text {rd }}$ term is for the thermal energy correction due to vibrational frequencies, and last term belongs to the entropy of the system. In case of $\mathrm{O}_{2}(\mathrm{~g})$ and $\mathrm{H}_{2}(\mathrm{~g})$ molecular free energy calculations, the thermal contributions are compressed into terms related to the enthalpy (Eq. S4) and entropy (Eq. S7). For example, for oxygen free energy can be calculated by these terms.

$$
\mu^{e l e}(O)=\frac{1}{2}\left[E_{O_{2}}+\frac{1}{2} \sum_{N=1}^{3(N-1)-3} h v_{i}+\int_{0}^{T} C_{p, O_{2}} T d T-T \Delta S_{O_{2}}\right]
$$

and similarly, for hydrogen free energy: 


$$
\mu^{e l e}(H)=\frac{1}{2}\left[E_{H_{2}}+\frac{1}{2} \sum_{N=1}^{3(N-1)-3} h v_{i}+\int_{0}^{T} C_{p, H_{2}} T d T-T \Delta S_{H_{2}}\right]
$$

In both the equations, first and second terms are the energies and zeropoint vibrational corrections of $\mathrm{O}_{2}$ and $\mathrm{H}_{2}$ molecules, respectively. Enthalpy is calculated by

$$
H(T)-H(0 K)=\frac{1}{2}\left(\int_{0}^{T} C_{p, O_{2}} T d T\right)=K_{B} T\left[\frac{7}{2}+\frac{\theta_{v}}{T} \frac{1}{e^{\frac{\theta_{v}}{T}}-1}\right]
$$

Since $\mathrm{O}_{2}$ and $\mathrm{H}_{2}$, both have 2 atoms in the molecules, third term in Eq. $\mathrm{S} 2$ and $\mathrm{S} 3$ is calculated similarly by Eq. S4.

In Eq. $\mathrm{S} 4, \mathrm{H}$ is the enthalpy, $\mathrm{R}$ is the gas constant, $\mathrm{T}$ is the absolute temperature and $\Theta_{\mathrm{v}}$ is the vibrational temperature. The factor of 7/2 arises from the ideal gas and rigid-rotor approximations for a homonuclear diatomic molecule. $\Theta_{\mathrm{v}}$ is defined where $v$ is the vibrational frequency, $\mathrm{h}$ is Planck's constant and kB is Boltzmann's constant.

$$
\Theta=\frac{h v}{k_{B}}
$$

Forth terms are the entropy terms, are calculated by-

$$
\begin{gathered}
s=s_{e}+s_{t}+s_{r}+s_{v} \\
=K_{B}\left[\ln \left(q_{e}\right)+\left(\ln \left(q_{t}\right)+\frac{5}{2}\right)+\left(\ln \left(q_{v}\right)+\frac{\theta_{v}}{T} \frac{1}{e^{\frac{\theta_{v}}{T}}-1}\right)+\left(\ln \left(q_{r}\right)+1\right)\right]
\end{gathered}
$$

Where,

$$
q_{t}=\left(\frac{2 \pi M K_{B} T}{h^{2}}\right)^{\frac{3}{2}} V ; q_{r}=\left(\frac{8 \pi^{2} I K_{B} T}{2 h^{2}}\right) ; q_{v}=\left(\frac{1}{1-e^{-\frac{h v}{k T}}}\right)
$$

and $\mathrm{q}_{\mathrm{e}}=3$ (triplet for $\mathrm{O}_{2}$ molecule) and 1 (singlet for $\mathrm{H}_{2}$ molecule), $\mathrm{I}=$ Moment of Inertia, $\mathrm{M}$ and $\mathrm{V}=$ mass and volume of the molecule. 

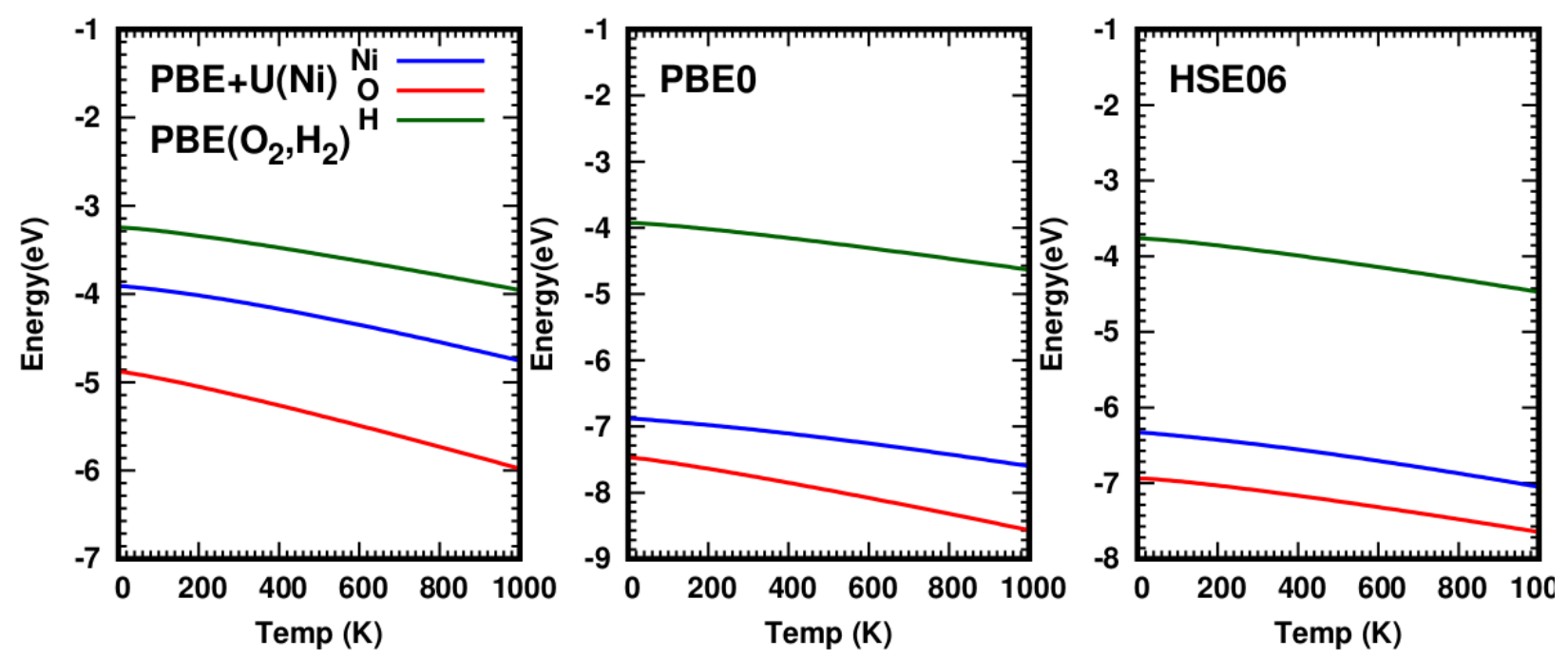

Figure S1: Variation in chemical potentials or elemental free energies with temperature after adding all the thermal and vibrational corrections of $\mathrm{Ni}(\mathrm{s}), \mathrm{O}(\mathrm{g})$, and $\mathrm{H}(\mathrm{g})$ atoms for

PBE+U/PBE, PBE0 and HSE06 functional.
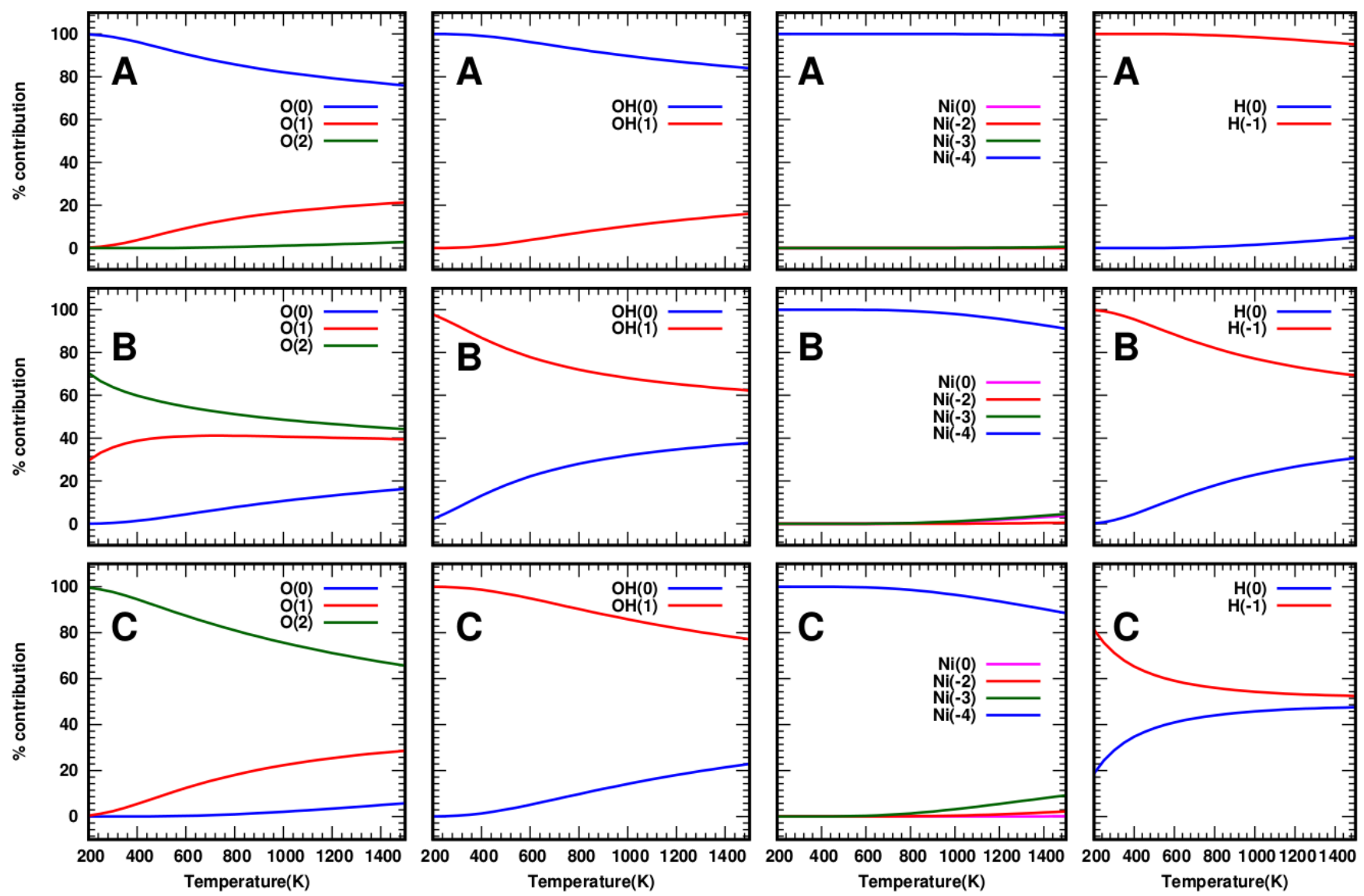
Figure S2: Percentage contribution of the charge vacancies considered for HSE06 functional.
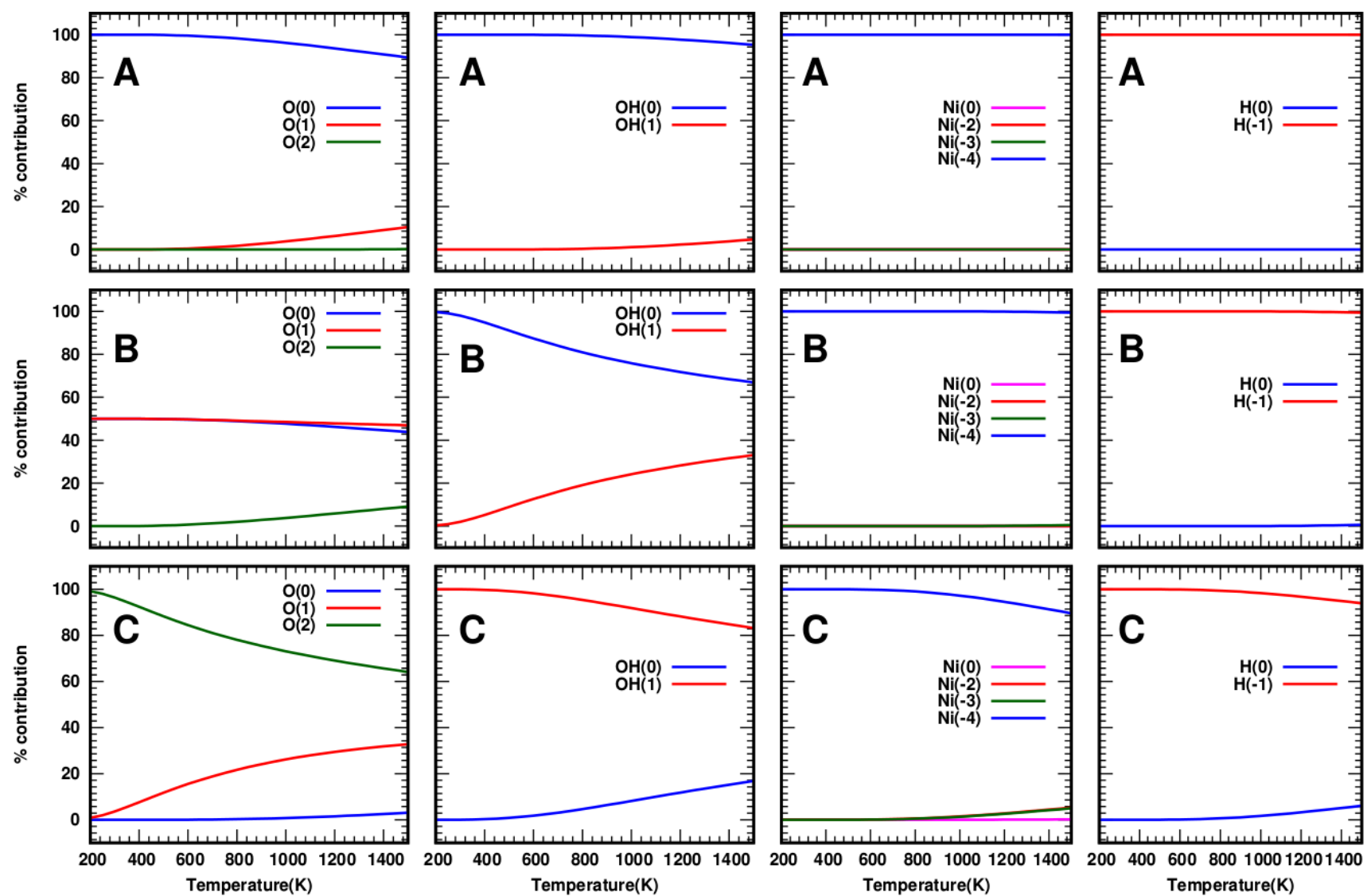

Figure S3: Percentage contribution of the charge vacancies considered for PBE0 functional. 


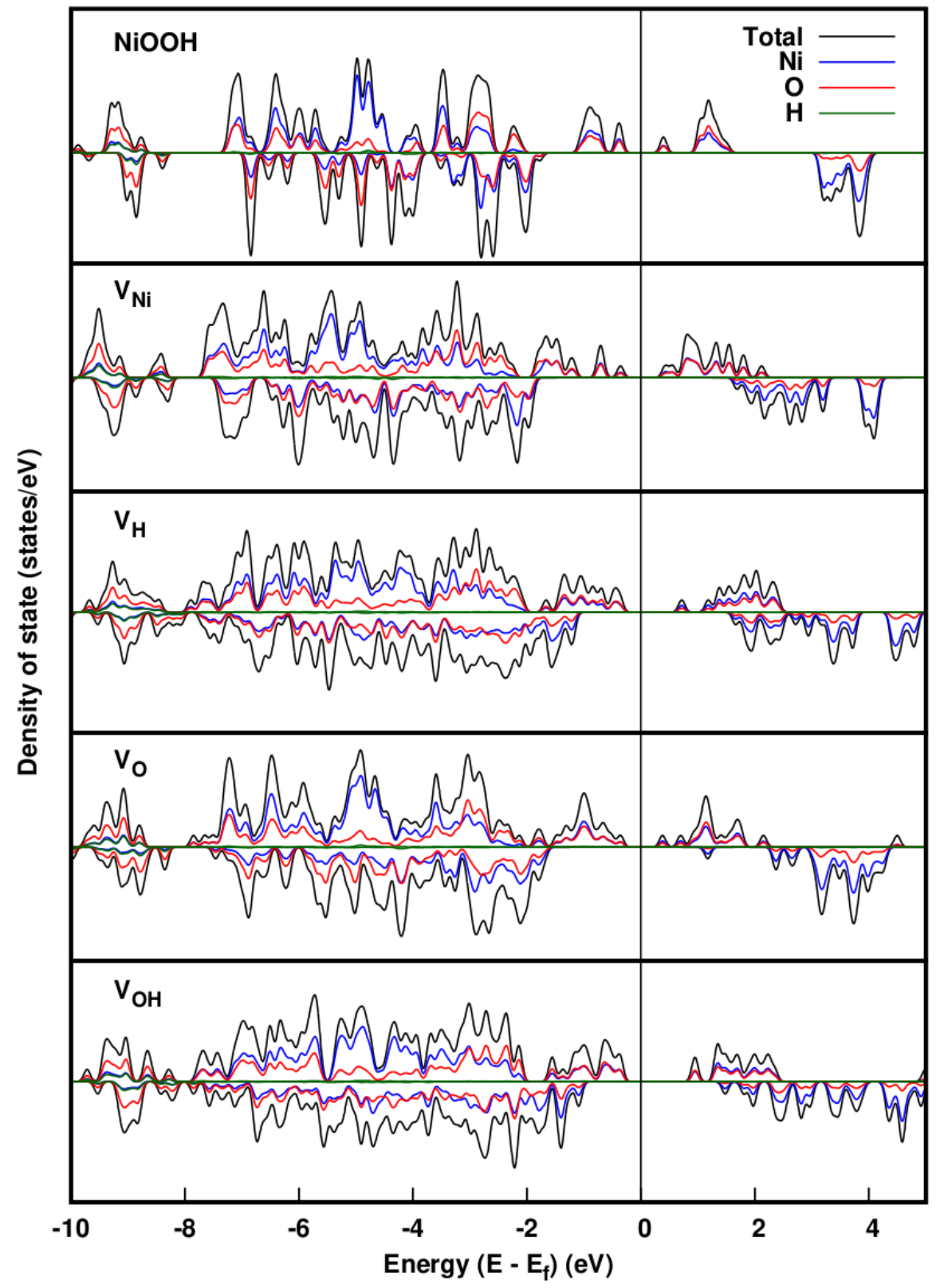

Figure S4: Projected density of state (PDOS) for pure $\mathrm{NiOOH}$ and with all the neutral vacancies considered in case of HSE06 functional. 


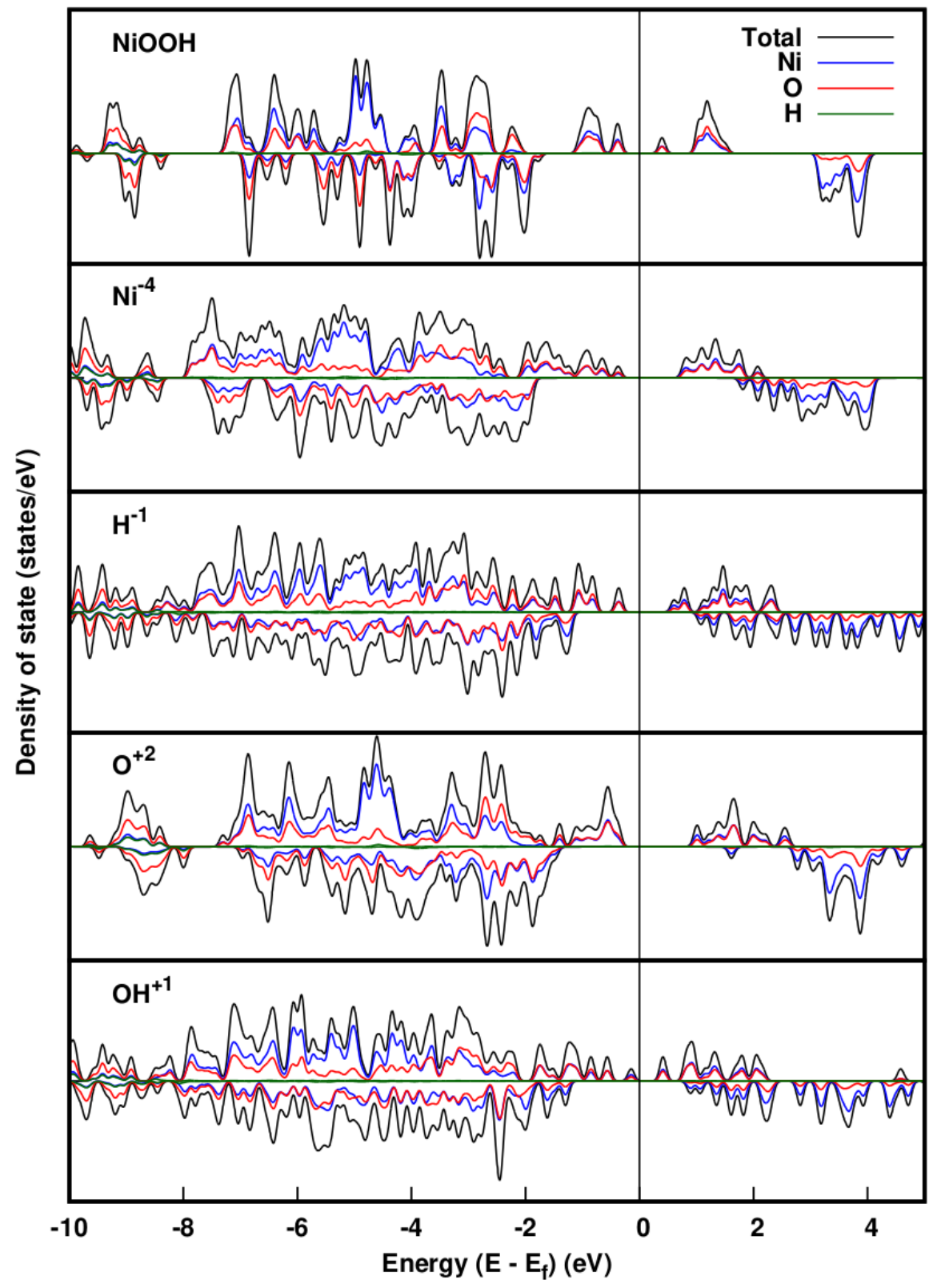

Figure S5: Projected density of state (PDOS) for pure $\mathrm{NiOOH}$ and with all the charged vacancies using HSE06 hybrid functional. 


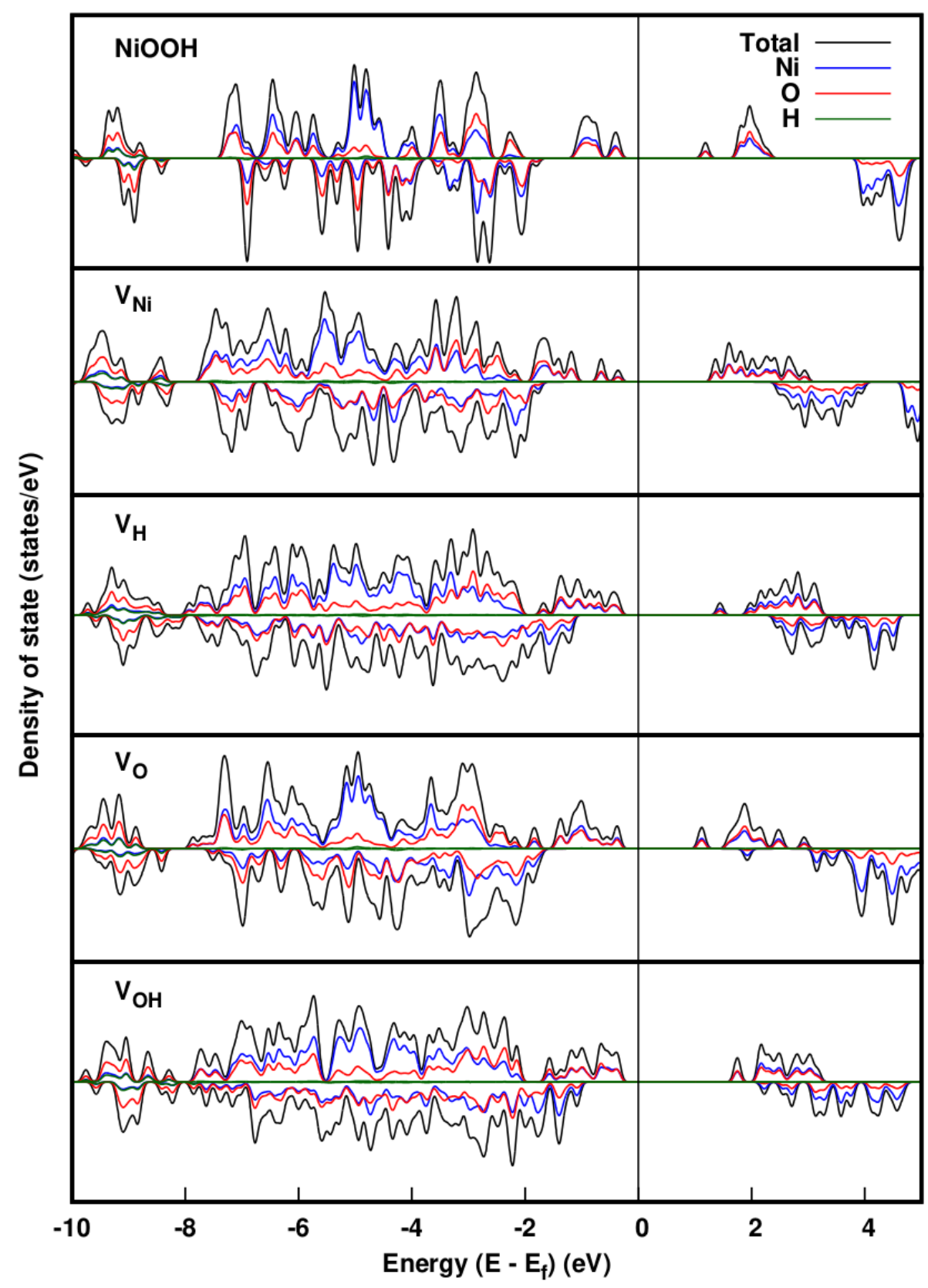

Figure S6: Projected density of state (PDOS) for pure $\mathrm{NiOOH}$ and with all the neutral vacancies considered in case of PBE0 hybrid functional. 


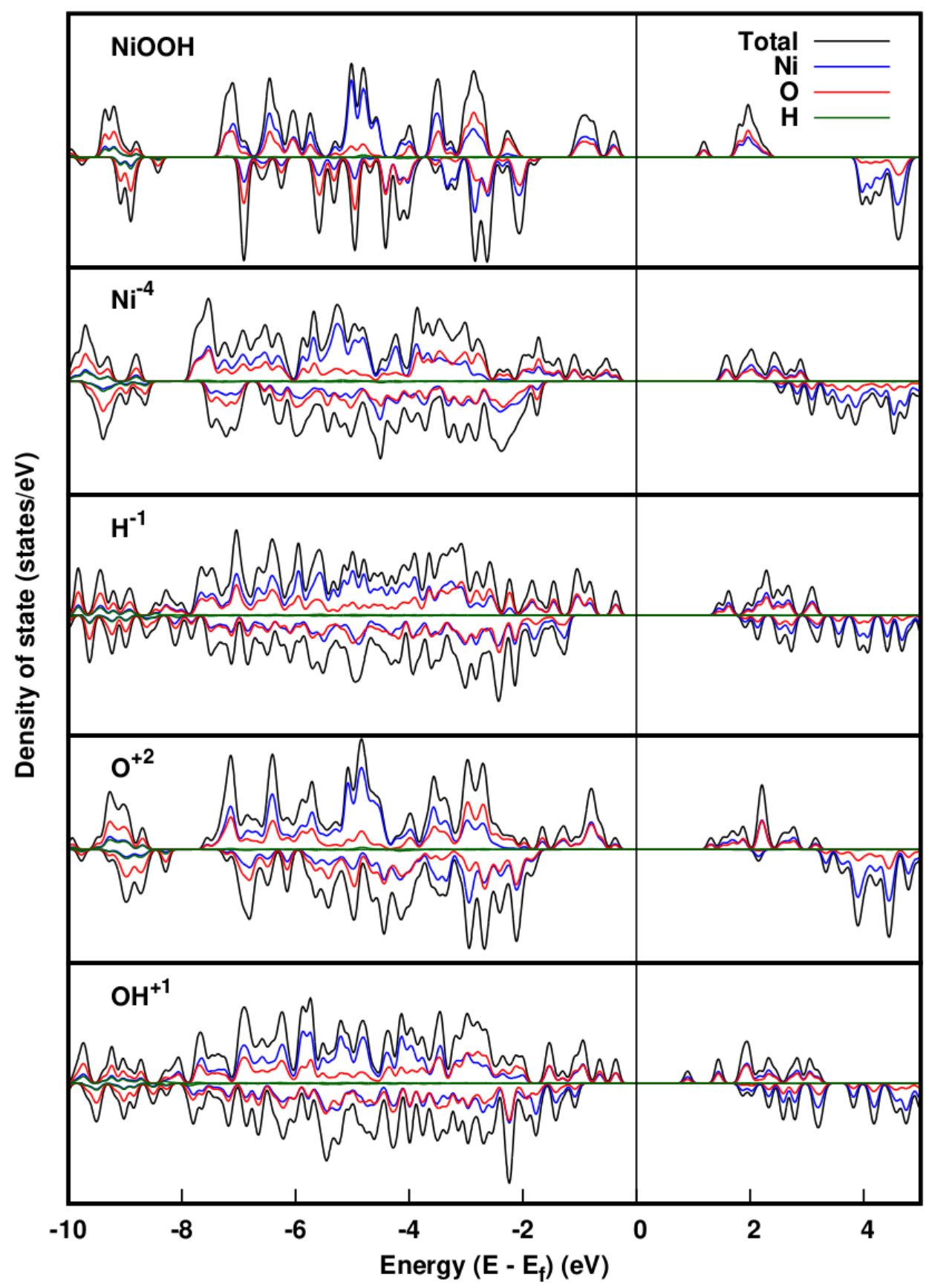

Figure S7: Projected density of state (PDOS) for pure $\mathrm{NiOOH}$ and with all the charged vacancies using PBE0 hybrid functional. 
Table S1: Evbm for $\beta$-NiOOH using hybrid PBE0 and HSE06 functionals.

\begin{tabular}{|c|c|}
\hline Functional & EvBM \\
\hline PBE0 & 2.52 \\
\hline HSE006 & 2.79 \\
\hline
\end{tabular}

In (Fig S8), we have plotted Ni neutral and charged vacancy formation energies with the function of Fermi energy (Ef). Initially at $\mathrm{Ef}=0$, neutral Ni vacancy is found lowest till $\mathrm{Ef}=0.12 \mathrm{eV}$, which is our transition level for $\mathrm{Ni}$ case as mentioned in Eq 3 and Table 4. After that $\mathrm{Ni}(-4)$ vacancy formation dominates. Transition $(0,-4),(0,-3)$ and $(0,-2)$ are very near to each other. Therefore, it is hard to make a strong comment about which charge state for Ni vacancy is more stable.

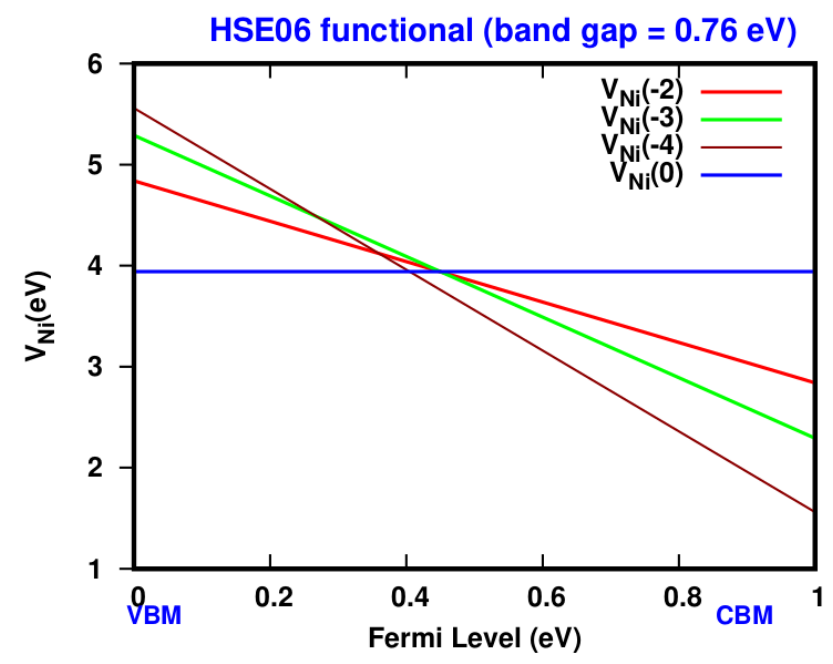

Figure S8: Ni vacancy formation vs. Fermi energy of $\beta$-NiOOH by using HSE06 functional.

\section{System size effect for charge defects:}

Generally, charged defect calculations subject to periodic boundary conditions are plagued by strong supercell-size-dependent errors caused by the interaction of the defect with its periodic images and the compensating background charge. In our charge defect calculations, there are mainly 2 corrections are added: First, a neutralization background jellium charge is introduced in order to avoid the divergence of electrostatic energy. This correction is already implemented in 
VASP package (https://cms.mpi.univie.ac.at/vasp /guide/node143.html). Second, the interaction of the resulting localized charges in the neutralizing background with respect to the supercell size is considered. As discussed in the computational detail section, $\Delta \mathrm{V}$ term is used to align the potential of bulk and defects. Here we demonstrate the size effect of converging energies by taking two different sized supercells. We have taken $2 \times 3 \times 2$ sized supercell and compare the results of formation energies with our main $3 \times 3 \times 2$ sized supercell. Eq. 2 is used to calculate, and results are shown in the Table S2. Formation energies are found close to each other which shows the conversance of energy in the presence of homogeneous background charge.

Table S2: Charged defect formation energies of oxygen with -2 charge state by using HSE06 functional (at $\mathrm{E}_{\mathrm{f}}=\mathbf{0}$ ).

\begin{tabular}{|l|c|l|l|l|l|l|l|}
\hline $\begin{array}{c}\text { Supercell } \\
\text { size }\end{array}$ & \# of Atoms & $\mathrm{E}_{\text {perfect }}(\mathrm{eV})$ & $\mathrm{E}_{\text {Defect }}\left(\mathrm{O}^{-2}\right)(\mathrm{eV})$ & $\mathrm{E}_{\text {ele }}(\mathrm{O})(\mathrm{eV})$ & $\Delta \mu(\mathrm{eV})$ & $\Delta \mathrm{V}(\mathrm{eV})$ & $\Delta \mathrm{H}_{\mathrm{f}}(\mathrm{eV})$ \\
\hline $2 \times 3 \times 2$ & 48 & -342.35 & -341.42 & -6.94 & -1.04 & 0.1497 & -1.04 \\
\hline $3 \times 3 \times 2$ & 72 & -508.82 & -508.22 & -6.94 & -1.04 & 0.0727 & -1.01 \\
\hline
\end{tabular}

\section{Cell Structures:}

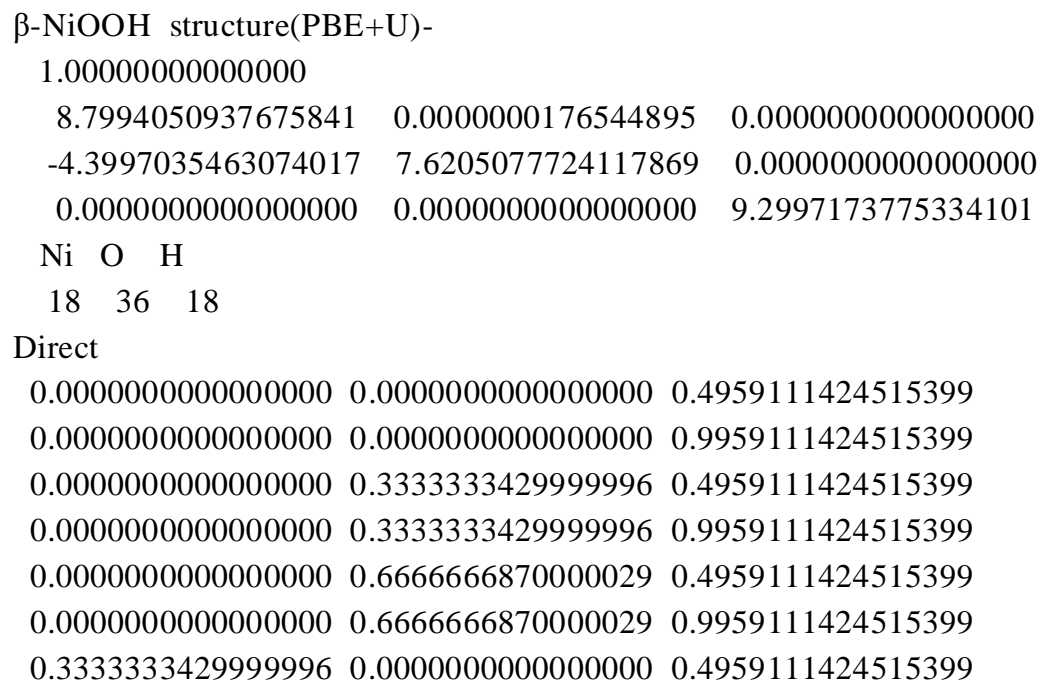


$\begin{array}{llll}0.3333333429999996 & 0.0000000000000000 & 0.9959111424515399\end{array}$ $\begin{array}{lllll}0.3333333429999996 & 0.3333333429999996 & 0.4959111424515399\end{array}$ $\begin{array}{lllll}0.3333333429999996 & 0.3333333429999996 & 0.9959111424515399\end{array}$ $\begin{array}{lllll}0.3333333429999996 & 0.6666666870000029 & 0.4959111424515399\end{array}$ $\begin{array}{llll}0.3333333429999996 & 0.6666666870000029 & 0.9959111424515399\end{array}$ $\begin{array}{llll}0.6666666870000029 & 0.0000000000000000 & 0.4959111424515399\end{array}$ $\begin{array}{llll}0.6666666870000029 & 0.0000000000000000 & 0.9959111424515399\end{array}$ $\begin{array}{llll}0.6666666870000029 & 0.3333333429999996 & 0.4959111424515399\end{array}$ $\begin{array}{llll}0.6666666870000029 & 0.3333333429999996 & 0.9959111424515399\end{array}$ $\begin{array}{llll}0.6666666870000029 & 0.6666666870000029 & 0.4959111424515399\end{array}$ $\begin{array}{llll}0.6666666870000029 & 0.6666666870000029 & 0.9959111424515399\end{array}$ $\begin{array}{llll}0.1111111120000032 & 0.2222223280000009 & 0.1012176036949697\end{array}$ $\begin{array}{llll}0.1111111120000032 & 0.2222223280000009 & 0.6012176266949680\end{array}$ $\begin{array}{llll}0.1111111120000032 & 0.5555556420000016 & 0.1012176036949697\end{array}$ $\begin{array}{llll}0.1111111120000032 & 0.5555556420000016 & 0.6012176266949680\end{array}$ $\begin{array}{llll}0.1111111120000032 & 0.8888890149999966 & 0.1012176036949697\end{array}$ $\begin{array}{llll}0.1111111120000032 & 0.8888890149999966 & 0.6012176266949680\end{array}$ $\begin{array}{lllll}0.4444444479999987 & 0.2222223280000009 & 0.1012176036949697\end{array}$ $\begin{array}{lllll}0.4444444479999987 & 0.2222223280000009 & 0.6012176266949680\end{array}$ $\begin{array}{lllll}0.4444444479999987 & 0.5555556420000016 & 0.1012176036949697\end{array}$ $\begin{array}{lllll}0.4444444479999987 & 0.5555556420000016 & 0.6012176266949680\end{array}$ $\begin{array}{lllll}0.4444444479999987 & 0.8888890149999966 & 0.1012176036949697\end{array}$ $\begin{array}{lllll}0.4444444479999987 & 0.8888890149999966 & 0.6012176266949680\end{array}$ $\begin{array}{lllll}0.7777777909999983 & 0.2222223280000009 & 0.1012176036949697\end{array}$ $\begin{array}{lllll}0.7777777909999983 & 0.2222223280000009 & 0.6012176266949680\end{array}$ $\begin{array}{lllll}0.7777777909999983 & 0.5555556420000016 & 0.1012176036949697\end{array}$ $\begin{array}{lllll}0.7777777909999983 & 0.5555556420000016 & 0.6012176266949680\end{array}$ $\begin{array}{lllll}0.7777777909999983 & 0.8888890149999966 & 0.1012176036949697\end{array}$ $\begin{array}{llll}0.7777777909999983 & 0.8888890149999966 & 0.6012176266949680\end{array}$ $\begin{array}{llll}0.2222223280000009 & 0.1111111120000032 & 0.3869035534902139\end{array}$ $\begin{array}{llll}0.2222223280000009 & 0.1111111120000032 & 0.8869035244902150\end{array}$ $\begin{array}{lllll}0.2222223280000009 & 0.4444444479999987 & 0.3869035534902139\end{array}$ $\begin{array}{lllll}0.2222223280000009 & 0.4444444479999987 & 0.8869035244902150\end{array}$ $\begin{array}{llll}0.2222223280000009 & 0.7777777909999983 & 0.3869035534902139\end{array}$ $\begin{array}{llll}0.2222223280000009 & 0.7777777909999983 & 0.8869035244902150\end{array}$ $\begin{array}{lllll}0.5555556420000016 & 0.1111111120000032 & 0.3869035534902139\end{array}$ $\begin{array}{lllll}0.5555556420000016 & 0.1111111120000032 & 0.8869035244902150\end{array}$ $\begin{array}{lllll}0.5555556420000016 & 0.4444444479999987 & 0.3869035534902139\end{array}$ $\begin{array}{lllll}0.5555556420000016 & 0.4444444479999987 & 0.8869035244902150\end{array}$ $\begin{array}{llll}0.5555556420000016 & 0.7777777909999983 & 0.3869035534902139\end{array}$ $\begin{array}{llll}0.5555556420000016 & 0.7777777909999983 & 0.8869035244902150\end{array}$ $\begin{array}{llll}0.8888890149999966 & 0.1111111120000032 & 0.3869035534902139\end{array}$ $\begin{array}{lllll}0.8888890149999966 & 0.1111111120000032 & 0.8869035244902150\end{array}$ $\begin{array}{lllll}0.8888890149999966 & 0.4444444479999987 & 0.3869035534902139\end{array}$ $\begin{array}{lllll}0.8888890149999966 & 0.4444444479999987 & 0.8869035244902150\end{array}$ $\begin{array}{lllll}0.8888890149999966 & 0.7777777909999983 & 0.3869035534902139\end{array}$ $\begin{array}{llll}0.8888890149999966 & 0.7777777909999983 & 0.8869035244902150\end{array}$ $\begin{array}{lllll}0.2222223280000009 & 0.1111109929999969 & 0.2809528213632788\end{array}$ $\begin{array}{llll}0.2222223280000009 & 0.1111109929999969 & 0.7809528513632813\end{array}$ 
$\begin{array}{llll}0.2222223280000009 & 0.4444443289999995 & 0.2809528213632788\end{array}$

$\begin{array}{llll}0.2222223280000009 & 0.4444443289999995 & 0.7809528513632813\end{array}$

$\begin{array}{lllll}0.2222223280000009 & 0.7777776719999991 & 0.2809528213632788\end{array}$

$\begin{array}{lllll}0.2222223280000009 & 0.7777776719999991 & 0.7809528513632813\end{array}$

$\begin{array}{llll}0.5555556420000016 & 0.1111109929999969 & 0.2809528213632788\end{array}$

$\begin{array}{lllll}0.5555556420000016 & 0.1111109929999969 & 0.7809528513632813\end{array}$

$\begin{array}{lllll}0.5555556420000016 & 0.4444443289999995 & 0.2809528213632788\end{array}$

$\begin{array}{llll}0.5555556420000016 & 0.4444443289999995 & 0.7809528513632813\end{array}$

$\begin{array}{lllll}0.5555556420000016 & 0.7777776719999991 & 0.2809528213632788\end{array}$

$\begin{array}{lllll}0.5555556420000016 & 0.7777776719999991 & 0.7809528513632813\end{array}$

$\begin{array}{lllll}0.8888890149999966 & 0.1111109929999969 & 0.2809528213632788\end{array}$

$\begin{array}{lllll}0.8888890149999966 & 0.1111109929999969 & 0.7809528513632813\end{array}$

$\begin{array}{lllll}0.8888890149999966 & 0.4444443289999995 & 0.2809528213632788\end{array}$

$\begin{array}{llll}0.8888890149999966 & 0.4444443289999995 & 0.7809528513632813\end{array}$

$\begin{array}{lllll}0.8888890149999966 & 0.7777776719999991 & 0.2809528213632788\end{array}$

$\begin{array}{lllll}0.8888890149999966 & 0.7777776719999991 & 0.7809528513632813\end{array}$

$\beta-\mathrm{NiOOH}$ (with $\mathrm{Ni}$ vacancy) structure $(\mathrm{PBE}+\mathrm{U})-$

1.00000000000000

$\begin{array}{rrr}8.7994050937675841 & 0.0000000176544895 & 0.0000000000000000 \\ -4.3997035463074017 & 7.6205077724117869 & 0.0000000000000000 \\ 0.0000000000000000 & 0.0000000000000000 & 9.2997173775334101\end{array}$

$\mathrm{Ni} \mathrm{O} \quad \mathrm{H}$

$\begin{array}{lll}17 \quad 36 & 18\end{array}$

Direct

$\begin{array}{llll}0.0014924602854975 & 0.0015876386899976 & 0.4967587518848262\end{array}$

$\begin{array}{llll}0.9997976174233543 & 0.0007217125662038 & 0.9931350098593086\end{array}$

$\begin{array}{lllll}0.0014924602854975 & 0.3332381645955138 & 0.4967587518848262\end{array}$

$\begin{array}{llll}0.9997976174233543 & 0.3324092478571430 & 0.9931350098593086\end{array}$

$\begin{array}{llll}0.0000000000000000 & 0.6666666870000029 & 0.4999128638831536\end{array}$

$\begin{array}{lllll}0.0000000000000000 & 0.6666666870000029 & 0.9975271671335193\end{array}$

$\begin{array}{lllll}0.3334285214044854 & 0.0015876386899976 & 0.4967587518848262\end{array}$

$\begin{array}{llll}0.3342574381428562 & 0.0007217125662038 & 0.9931350098593086\end{array}$

$\begin{array}{lllll}0.3333333429999996 & 0.3333333429999996 & 0.0009130110404172\end{array}$

$\begin{array}{lllll}0.3334285214044854 & 0.6651742267144982 & 0.4967587518848262\end{array}$

$\begin{array}{llll}0.3342574381428562 & 0.6668690695766486 & 0.9931350098593086\end{array}$

$\begin{array}{lllll}0.6666666870000029 & 0.0000000000000000 & 0.4925793553080808\end{array}$

$\begin{array}{lllll}0.6666666870000029 & 0.0000000000000000 & 0.9860372950080887\end{array}$

$\begin{array}{llll}0.6650790483100053 & 0.3332381645955138 & 0.4967587518848262\end{array}$

$\begin{array}{lllll}0.6659449744337991 & 0.3324092478571430 & 0.9931350098593086\end{array}$

$\begin{array}{llll}0.6650790483100053 & 0.6651742267144982 & 0.4967587518848262\end{array}$ $\begin{array}{llll}0.6659449744337991 & 0.6668690695766486 & 0.9931350098593086\end{array}$ $\begin{array}{llll}0.0998437471949885 & 0.2165886455974970 & 0.1003745111128822\end{array}$ $\begin{array}{llll}0.0646349678965450 & 0.1989842559482753 & 0.6152445397866728\end{array}$ $\begin{array}{llll}0.1151894087570327 & 0.5514773452429722 & 0.1008449795338535\end{array}$ $\begin{array}{lllll}0.1160188508711997 & 0.5506479031288052 & 0.6021888775389641\end{array}$ $\begin{array}{llll}0.1151894087570327 & 0.8970456085140555 & 0.1008449795338535\end{array}$ $\begin{array}{llll}0.1160188508711997 & 0.8987044927423966 & 0.6021888775389641\end{array}$ $\begin{array}{llll}0.4500781304025026 & 0.2165886455974970 & 0.1003745111128822\end{array}$ 
$\begin{array}{llll}0.4676825200517243 & 0.1989842559482753 & 0.6152445397866728\end{array}$ $\begin{array}{llll}0.4500781304025026 & 0.5668230068050164 & 0.1003745111128822\end{array}$ $\begin{array}{llll}0.4676825200517243 & 0.6020317861034599 & 0.6152445397866728\end{array}$ $\begin{array}{llll}0.4411243299235608 & 0.8872289559617812 & 0.0950903130344400\end{array}$ $\begin{array}{llll}0.4316211754907826 & 0.8824773787453850 & 0.5949773728502734\end{array}$ $\begin{array}{llll}0.7794378500382138 & 0.2255424460764388 & 0.0950903130344400\end{array}$ $\begin{array}{llll}0.7841894272546099 & 0.2350456005092170 & 0.5949773728502734\end{array}$ $\begin{array}{llll}0.7696211974859395 & 0.5514773452429722 & 0.1008449795338535\end{array}$ $\begin{array}{llll}0.7679623132575983 & 0.5506479031288052 & 0.6021888775389641\end{array}$ $\begin{array}{llll}0.7794378500382138 & 0.8872289559617812 & 0.0950903130344400\end{array}$ $\begin{array}{llll}0.7841894272546099 & 0.8824773787453850 & 0.5949773728502734\end{array}$ $\begin{array}{lllll}0.2200111269489113 & 0.1066887098978171 & 0.3970557332771278\end{array}$ $\begin{array}{lllll}0.2213443883127511 & 0.1093552326255107 & 0.8858507423535684\end{array}$ $\begin{array}{llll}0.2200111269489113 & 0.4466556490510882 & 0.3970557332771278\end{array}$ $\begin{array}{lllll}0.2213443883127511 & 0.4453223876872485 & 0.8858507423535684\end{array}$ $\begin{array}{lllll}0.2250379611470308 & 0.7791856075735097 & 0.3871497603286969\end{array}$ $\begin{array}{llll}0.2250819998859868 & 0.7792076269429913 & 0.8853657672546689\end{array}$ $\begin{array}{lllll}0.5510469712295532 & 0.1156197827704517 & 0.3844293964686969\end{array}$ $\begin{array}{lllll}0.5516110938519958 & 0.1150556601480091 & 0.8797337876330573\end{array}$ $\begin{array}{lllll}0.5599780441021878 & 0.4466556490510882 & 0.3970557332771278\end{array}$ $\begin{array}{llll}0.5573115213744870 & 0.4453223876872485 & 0.8858507423535684\end{array}$ $\begin{array}{llll}0.5510469712295532 & 0.7687604494591014 & 0.3844293964686969\end{array}$ $\begin{array}{llll}0.5516110938519958 & 0.7698886947039796 & 0.8797337876330573\end{array}$ $\begin{array}{lllll}0.8979063565408936 & 0.1156197827704517 & 0.3844293964686969\end{array}$ $\begin{array}{lllll}0.8967781112960154 & 0.1150556601480091 & 0.8797337876330573\end{array}$ $\begin{array}{llll}0.8874811984264852 & 0.4416288148529688 & 0.3871497603286969\end{array}$ $\begin{array}{llll}0.8874591790570037 & 0.4415847761140128 & 0.8853657672546689\end{array}$ $\begin{array}{lllll}0.8874811984264852 & 0.7791856075735097 & 0.3871497603286969\end{array}$ $\begin{array}{llll}0.8874591790570037 & 0.7792076269429913 & 0.8853657672546689\end{array}$ $\begin{array}{lllll}0.1993310247940769 & 0.0653283865881491 & 0.2961181053215896\end{array}$ $\begin{array}{lllll}0.2181162809268571 & 0.1028988988537165 & 0.7798975648981923\end{array}$ $\begin{array}{llll}0.1993310247940769 & 0.4673356322059234 & 0.2961181053215896\end{array}$ $\begin{array}{lllll}0.2181162809268571 & 0.4485503760731433 & 0.7798975648981923\end{array}$ $\begin{array}{lllll}0.2354169275179956 & 0.7843749717589930 & 0.2814876898201675\end{array}$ $\begin{array}{lllll}0.2352971005965188 & 0.7843150582982616 & 0.7797033105055391\end{array}$ $\begin{array}{lllll}0.5401588849516799 & 0.1265077500483187 & 0.2794993614174643\end{array}$ $\begin{array}{llll}0.5365671589960428 & 0.1300994760039558 & 0.7741511831009404\end{array}$ $\begin{array}{lllll}0.6013382484118495 & 0.4673356322059234 & 0.2961181053215896\end{array}$ $\begin{array}{lllll}0.5637677361462821 & 0.4485503760731433 & 0.7798975648981923\end{array}$ $\begin{array}{llll}0.5401588849516799 & 0.7469841579033627 & 0.2794993614174643\end{array}$ $\begin{array}{lllll}0.5365671589960428 & 0.7398007059920744 & 0.7741511831009404\end{array}$ $\begin{array}{lllll}0.9196825290966331 & 0.1265077500483187 & 0.2794993614174643\end{array}$ $\begin{array}{llll}0.9268659810079214 & 0.1300994760039558 & 0.7741511831009404\end{array}$ $\begin{array}{lllll}0.8822917152410028 & 0.4312497294820048 & 0.2814876898201675\end{array}$ $\begin{array}{lllll}0.8823516287017341 & 0.4313695564034816 & 0.7797033105055391\end{array}$ $\begin{array}{lllll}0.8822917152410028 & 0.7843749717589930 & 0.2814876898201675\end{array}$ $\begin{array}{llll}0.8823516287017341 & 0.7843150582982616 & 0.7797033105055391\end{array}$

$\beta-\mathrm{NiOOH}$ (with O vacancy) structure $(\mathrm{PBE}+\mathrm{U})-$ 
1.00000000000000

$\begin{array}{lll}8.7994050937675841 & 0.0000000176544895 & 0.0000000000000000 \\ -4.3997035463074017 & 7.6205077724117869 & 0.0000000000000000 \\ 0.0000000000000000 & 0.0000000000000000 & 9.2997173775334101 \\ \text { Ni O H } & & \end{array}$

$\begin{array}{lll}18 & 35 & 18\end{array}$

Direct

$\begin{array}{llll}0.0010042346578416 & 0.0005021173289208 & 0.5017561373917943\end{array}$

$\begin{array}{llll}0.9997438143638036 & 0.9998719071819053 & 0.9984628590186162\end{array}$

$\begin{array}{lllll}0.0007416984616455 & 0.3348167399232906 & 0.4937389761579496\end{array}$

$\begin{array}{llll}0.9999693824359284 & 0.3332721078718706 & 0.9934154793493590\end{array}$

$\begin{array}{lllll}0.0007416984616455 & 0.6659249885383574 & 0.4937389761579496\end{array}$

$\begin{array}{llll}0.9999693824359284 & 0.6666973045640745 & 0.9934154793493590\end{array}$

$\begin{array}{llll}0.3269325941713745 & 0.9871985023427499 & 0.4905634937543581\end{array}$

$\begin{array}{llll}0.3328331514031504 & 0.9989996168063016 & 0.9948745453620944\end{array}$

$\begin{array}{lllll}0.3269325941713745 & 0.3397340918286247 & 0.4905634937543581\end{array}$

$\begin{array}{lllll}0.3328331514031504 & 0.3338335345968488 & 0.9948745453620944\end{array}$

$\begin{array}{lllll}0.3318499460767086 & 0.6659249885383574 & 0.4937389761579496\end{array}$

$\begin{array}{lllll}0.3333945781281287 & 0.6666973045640745 & 0.9934154793493590\end{array}$

$\begin{array}{lllll}0.6661645696710821 & 0.0005021173289208 & 0.5017561373917943\end{array}$

$\begin{array}{llll}0.6667947798180975 & 0.9998719071819053 & 0.9984628590186162\end{array}$

$\begin{array}{lllll}0.6794681846572530 & 0.3397340918286247 & 0.4905634937543581\end{array}$

$\begin{array}{lllll}0.6676670701937013 & 0.3338335345968488 & 0.9948745453620944\end{array}$

$\begin{array}{lllll}0.6661645696710821 & 0.6656624523421613 & 0.5017561373917943\end{array}$

$\begin{array}{lllll}0.6667947798180975 & 0.6669228726361922 & 0.9984628590186162\end{array}$

$\begin{array}{llll}0.1102033910731990 & 0.2233592923173333 & 0.1008505048697117\end{array}$

$\begin{array}{lllll}0.1203598493564684 & 0.2264301079812086 & 0.6034732069676423\end{array}$

$\begin{array}{lllll}0.1111111120000032 & 0.5555556420000016 & 0.0976298773265896\end{array}$

$\begin{array}{lllll}0.1111111120000032 & 0.5555556420000016 & 0.5978656609587816\end{array}$

$\begin{array}{llll}0.1102033910731990 & 0.8868443297558599 & 0.1008505048697117\end{array}$

$\begin{array}{llllll}0.1203598493564684 & 0.8939299723752541 & 0.6034732069676423\end{array}$

$\begin{array}{lllll}0.4444444479999987 & 0.2222223280000009 & 0.1016252035820884\end{array}$

$\begin{array}{llll}0.4433074836826663 & 0.5564633629268059 & 0.1008505048697117\end{array}$

$\begin{array}{llll}0.4402366680187910 & 0.5463069046435365 & 0.6034732069676423\end{array}$

$\begin{array}{lllll}0.4433074836826663 & 0.8868443297558599 & 0.1008505048697117\end{array}$

$\begin{array}{lllll}0.4402366680187910 & 0.8939299723752541 & 0.6034732069676423\end{array}$

$\begin{array}{llll}0.7798224762441350 & 0.2233592923173333 & 0.1008505048697117\end{array}$

$\begin{array}{lllll}0.7727368336247409 & 0.2264301079812086 & 0.6034732069676423\end{array}$

$\begin{array}{lllll}0.7798224762441350 & 0.5564633629268059 & 0.1008505048697117\end{array}$

$\begin{array}{lllll}0.7727368336247409 & 0.5463069046435365 & 0.6034732069676423\end{array}$

$\begin{array}{lllll}0.7777777909999983 & 0.8888890149999966 & 0.1036001357441236\end{array}$

$\begin{array}{lllll}0.7777777909999983 & 0.8888890149999966 & 0.6115773971727450\end{array}$

$\begin{array}{lllll}0.2317076933833420 & 0.1158537946916738 & 0.3927842843070053\end{array}$

$\begin{array}{lllll}0.2194166217351849 & 0.1097082588675917 & 0.8855360218020962\end{array}$

$\begin{array}{lllll}0.2194603999364944 & 0.4472063760635052 & 0.3774155341425427\end{array}$

$\begin{array}{lllll}0.2222719278414189 & 0.4443948481585807 & 0.8844197788912922\end{array}$

$\begin{array}{lllll}0.2194603999364944 & 0.7722539348729853 & 0.3774155341425427\end{array}$

$\begin{array}{lllll}0.2222719278414189 & 0.7778769906828344 & 0.8844197788912922\end{array}$

$\begin{array}{lllll}0.5508129593083311 & 0.1158537946916738 & 0.3927842843070053\end{array}$ 
$\begin{array}{lllll}0.5569584951324131 & 0.1097082588675917 & 0.8855360218020962\end{array}$ $\begin{array}{lllll}0.5508129593083311 & 0.4349590826166576 & 0.3927842843070053\end{array}$ $\begin{array}{llll}0.5569584951324131 & 0.4472501542648146 & 0.8855360218020962\end{array}$ $\begin{array}{llll}0.5561909597217252 & 0.7780954498608565 & 0.3915167059764642\end{array}$ $\begin{array}{lllll}0.5571623828274070 & 0.7785811614136975 & 0.8872954694093238\end{array}$ $\begin{array}{llll}0.8885713561391384 & 0.1104757942782797 & 0.3915167059764642\end{array}$ $\begin{array}{lllll}0.8880856445862975 & 0.1095043711726049 & 0.8872954694093238\end{array}$ $\begin{array}{llll}0.8944128711270096 & 0.4472063760635052 & 0.3774155341425427\end{array}$ $\begin{array}{llll}0.8887898153171605 & 0.4443948481585807 & 0.8844197788912922\end{array}$ $\begin{array}{lllll}0.8885713561391384 & 0.7780954498608565 & 0.3915167059764642\end{array}$ $\begin{array}{lllll}0.8880856445862975 & 0.7785811614136975 & 0.8872954694093238\end{array}$ $\begin{array}{lllll}0.2424630478585996 & 0.1212313529292928 & 0.2870345693374645\end{array}$ $\begin{array}{lllll}0.1999214120351027 & 0.0999605350175514 & 0.7807690353478947\end{array}$ $\begin{array}{lllll}0.2184245803123588 & 0.4482420766876416 & 0.2718658157786678\end{array}$ $\begin{array}{llll}0.2237716057832628 & 0.4428950512167376 & 0.7783157049307761\end{array}$ $\begin{array}{llll}0.2184245803123588 & 0.7701821766247221 & 0.2718658157786678\end{array}$ $\begin{array}{lllll}0.2237716057832628 & 0.7808762275665302 & 0.7783157049307761\end{array}$ $\begin{array}{llll}0.5454352820707058 & 0.1212313529292928 & 0.2870345693374645\end{array}$ $\begin{array}{lllll}0.5667060999824471 & 0.0999605350175514 & 0.7807690353478947\end{array}$ $\begin{array}{lllll}0.5454352820707058 & 0.4242036091414008 & 0.2870345693374645\end{array}$ $\begin{array}{lllll}0.5667060999824471 & 0.4667452449648977 & 0.7807690353478947\end{array}$ $\begin{array}{llll}0.5572328725164368 & 0.7786162872582238 & 0.2856147948052126\end{array}$ $\begin{array}{lllll}0.5635648542944764 & 0.7817822781472401 & 0.7813788680693037\end{array}$ $\begin{array}{lllll}0.8880503997417719 & 0.1094337624835617 & 0.2856147948052126\end{array}$ $\begin{array}{lllll}0.8848844088527557 & 0.1031017807055221 & 0.7813788680693037\end{array}$ $\begin{array}{lllll}0.8964845103752737 & 0.4482420766876416 & 0.2718658157786678\end{array}$ $\begin{array}{llll}0.8857904594334656 & 0.4428950512167376 & 0.7783157049307761\end{array}$ $\begin{array}{lllll}0.8880503997417719 & 0.7786162872582238 & 0.2856147948052126\end{array}$ $\begin{array}{llll}0.8848844088527557 & 0.7817822781472401 & 0.7813788680693037\end{array}$

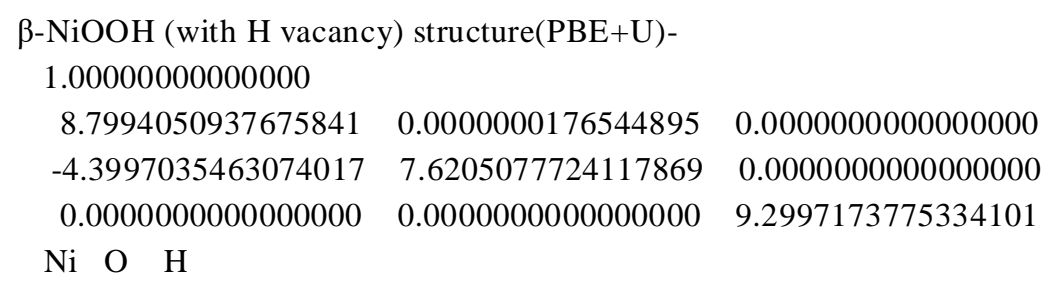
$\begin{array}{lll}18 & 36 & 17\end{array}$

Direct

$\begin{array}{llll}0.9962662684365640 & 0.0037337315634360 & 0.4980819259112579\end{array}$ $\begin{array}{llll}0.0001999511522470 & 0.9998000488477530 & 0.9997741008212984\end{array}$ $\begin{array}{llll}0.9962662684365640 & 0.3258658798731275 & 0.4980819259112579\end{array}$ $\begin{array}{llll}0.0001999511522470 & 0.3337332453045008 & 0.9997741008212984\end{array}$ $\begin{array}{lllll}0.9990170477514440 & 0.6661752108757284 & 0.4959567585186662\end{array}$ $\begin{array}{llll}0.9996528634427335 & 0.6664931187213696 & 0.9966257194289128\end{array}$ $\begin{array}{lllll}0.3338248191242741 & 0.0009829522485560 & 0.4959567585186662\end{array}$ $\begin{array}{llll}0.3335069112786329 & 0.0003471365572665 & 0.9966257194289128\end{array}$ $\begin{array}{llll}0.3328714990755586 & 0.3331024210377862 & 0.4939111668539482\end{array}$ 
$\begin{array}{llll}0.3343472206901978 & 0.3338402818451058 & 0.9918294282612479\end{array}$ $\begin{array}{lllll}0.3338248191242741 & 0.6661752108757284 & 0.4959567585186662\end{array}$ $\begin{array}{llll}0.3335069112786329 & 0.6664931187213696 & 0.9966257194289128\end{array}$ $\begin{array}{llll}0.6741341501268749 & 0.0037337315634360 & 0.4980819259112579\end{array}$ $\begin{array}{llll}0.6662667846955017 & 0.9998000488477530 & 0.9997741008212984\end{array}$ $\begin{array}{llll}0.6668976089622163 & 0.3331024210377862 & 0.4939111668539482\end{array}$ $\begin{array}{llll}0.6661597481548966 & 0.3338402818451058 & 0.9918294282612479\end{array}$ $\begin{array}{lllll}0.6668976089622163 & 0.6671285309244439 & 0.4939111668539482\end{array}$ $\begin{array}{lllll}0.6661597481548966 & 0.6656528093098046 & 0.9918294282612479\end{array}$ $\begin{array}{llll}0.1202708450778260 & 0.2268021945389123 & 0.1033885784641626\end{array}$ $\begin{array}{llll}0.1048968915663622 & 0.2191152177831768 & 0.6018743539605040\end{array}$ $\begin{array}{llll}0.1116022704842834 & 0.5565379589685691 & 0.1023655014331695\end{array}$ $\begin{array}{llll}0.1031284539618937 & 0.5395903259237826 & 0.6012447915530146\end{array}$ $\begin{array}{llll}0.1116022704842834 & 0.8883978565157165 & 0.1023655014331695\end{array}$ $\begin{array}{llll}0.1031284539618937 & 0.8968716730381061 & 0.6012447915530146\end{array}$ $\begin{array}{lllll}0.4467556914375734 & 0.2268448148751574 & 0.0972251626890852\end{array}$ $\begin{array}{lllll}0.4458958203902057 & 0.2251250727804077 & 0.5979998508380291\end{array}$ $\begin{array}{llll}0.4467556914375734 & 0.5532443985624269 & 0.0972251626890852\end{array}$ $\begin{array}{lllll}0.4458958203902057 & 0.5541042696097946 & 0.5979998508380291\end{array}$ $\begin{array}{llll}0.4434621310314313 & 0.8883978565157165 & 0.1023655014331695\end{array}$ $\begin{array}{llll}0.4604097640762177 & 0.8968716730381061 & 0.6012447915530146\end{array}$ $\begin{array}{llll}0.7731979244610869 & 0.2268021945389123 & 0.1033885784641626\end{array}$ $\begin{array}{llll}0.7808849012168224 & 0.2191152177831768 & 0.6018743539605040\end{array}$ $\begin{array}{llll}0.7731553041248418 & 0.5532443985624269 & 0.0972251626890852\end{array}$ $\begin{array}{lllll}0.7748750462195915 & 0.5541042696097946 & 0.5979998508380291\end{array}$ $\begin{array}{llll}0.7731979244610869 & 0.8797292819221738 & 0.1033885784641626\end{array}$ $\begin{array}{llll}0.7808849012168224 & 0.8951032354336448 & 0.6018743539605040\end{array}$ $\begin{array}{llll}0.2134395398748410 & 0.1104505347273772 & 0.3873274649821212\end{array}$ $\begin{array}{llll}0.2219684701736071 & 0.1107027724570173 & 0.8863492768669374\end{array}$ $\begin{array}{llll}0.2134395398748410 & 0.4363222371474649 & 0.3873274649821212\end{array}$ $\begin{array}{lllll}0.2219684701736071 & 0.4445989297165909 & 0.8863492768669374\end{array}$ $\begin{array}{llll}0.2222223280000009 & 0.7777777909999983 & 0.3903849785335112\end{array}$ $\begin{array}{llll}0.2222223280000009 & 0.7777777909999983 & 0.8864873836734475\end{array}$ $\begin{array}{llll}0.5636778528525355 & 0.1104505347273772 & 0.3873274649821212\end{array}$ $\begin{array}{llll}0.5554011602834095 & 0.1107027724570173 & 0.8863492768669374\end{array}$ $\begin{array}{lllll}0.5555556420000016 & 0.4444444479999987 & 0.3807382505262069\end{array}$ $\begin{array}{llll}0.5555556420000016 & 0.4444444479999987 & 0.8813814902196242\end{array}$ $\begin{array}{llll}0.5636778528525355 & 0.7865605791251582 & 0.3873274649821212\end{array}$ $\begin{array}{llll}0.5554011602834095 & 0.7780316488263921 & 0.8863492768669374\end{array}$ $\begin{array}{llll}0.8888890149999966 & 0.1111111120000032 & 0.3988159853719964\end{array}$ $\begin{array}{llll}0.8888890149999966 & 0.1111111120000032 & 0.8924135458911167\end{array}$ $\begin{array}{llll}0.8895495922726226 & 0.4363222371474649 & 0.3873274649821212\end{array}$ $\begin{array}{llll}0.8892973545429825 & 0.4445989297165909 & 0.8863492768669374\end{array}$ $\begin{array}{llll}0.8895495922726226 & 0.7865605791251582 & 0.3873274649821212\end{array}$ $\begin{array}{lllll}0.8892973545429825 & 0.7780316488263921 & 0.8863492768669374\end{array}$ $\begin{array}{lllll}0.2013985620386975 & 0.1131309578638096 & 0.2819147669904751\end{array}$ $\begin{array}{llll}0.2203112825467741 & 0.1063477094603158 & 0.7804167721152311\end{array}$ $\begin{array}{lllll}0.2013985620386975 & 0.4216005981748836 & 0.2819147669904751\end{array}$ $\begin{array}{llll}0.2203112825467741 & 0.4472965670864468 & 0.7804167721152311\end{array}$ 
$\begin{array}{llll}0.2222223280000009 & 0.7777776719999991 & 0.2844272946603468\end{array}$

$\begin{array}{lllll}0.2222223280000009 & 0.7777776719999991 & 0.7808323782665596\end{array}$

$\begin{array}{lllll}0.5783993728251176 & 0.1131309578638096 & 0.2819147669904751\end{array}$

$\begin{array}{lllll}0.5527034039135543 & 0.1063477094603158 & 0.7804167721152311\end{array}$

$\begin{array}{llll}0.5555556420000016 & 0.4444443289999995 & 0.2747162808042347\end{array}$

$\begin{array}{lllll}0.5555556420000016 & 0.4444443289999995 & 0.7752132142637933\end{array}$

$\begin{array}{llll}0.5783993728251176 & 0.7986014379613025 & 0.2819147669904751\end{array}$

$\begin{array}{llll}0.5527034039135543 & 0.7796887174532259 & 0.7804167721152311\end{array}$

$\begin{array}{lllll}0.8888890149999966 & 0.1111109929999969 & 0.7864870684974861\end{array}$

$\begin{array}{lllll}0.8868690501361840 & 0.4216005981748836 & 0.2819147669904751\end{array}$

$\begin{array}{llll}0.8936522985396778 & 0.4472965670864468 & 0.7804167721152311\end{array}$

$\begin{array}{llll}0.8868690501361840 & 0.7986014379613025 & 0.2819147669904751\end{array}$

$\begin{array}{llll}0.8936522985396778 & 0.7796887174532259 & 0.7804167721152311\end{array}$

$\beta-\mathrm{NiOOH}$ (with $\mathrm{OH}$ vacancy) structure $(\mathrm{PBE}+\mathrm{U})-$

1.00000000000000

$\begin{array}{rrr}8.7994050937675841 & 0.0000000176544895 & 0.0000000000000000 \\ -4.3997035463074017 & 7.6205077724117869 & 0.0000000000000000 \\ 0.0000000000000000 & 0.0000000000000000 & 9.2997173775334101\end{array}$

$\mathrm{Ni} \quad \mathrm{O} \quad \mathrm{H}$

$\begin{array}{lll}18 & 35 & 17\end{array}$

Direct

$\begin{array}{llll}0.0000927920056029 & 0.0000463960028014 & 0.4976392164841243\end{array}$

$\begin{array}{llll}0.9927972084082342 & 0.9963986042041171 & 0.0000727012169577\end{array}$

$\begin{array}{llll}0.9997768160451770 & 0.3335565269548226 & 0.4949759688305448\end{array}$

$\begin{array}{lllll}0.0004980980473661 & 0.3328352449526335 & 0.9954108739625269\end{array}$

$\begin{array}{lllll}0.9997768160451770 & 0.6662203190903497 & 0.4949759688305448\end{array}$

$\begin{array}{llll}0.0004980980473661 & 0.6676628830947280 & 0.9954108739625269\end{array}$

$\begin{array}{llll}0.3332869469971982 & 0.0000463960028014 & 0.4976392164841243\end{array}$

$\begin{array}{llll}0.3369347387958825 & 0.9963986042041171 & 0.0000727012169577\end{array}$

$\begin{array}{llll}0.3332869469971982 & 0.3332405509943968 & 0.4976392164841243\end{array}$

$\begin{array}{lllll}0.3369347387958825 & 0.3405361345917655 & 0.0000727012169577\end{array}$

$\begin{array}{llll}0.3331978107366922 & 0.6665989208683527 & 0.4968837401810333\end{array}$

$\begin{array}{llll}0.3351471012575260 & 0.6675735661287732 & 0.9963482891271696\end{array}$

$\begin{array}{llll}0.6667344531316530 & 0.0001355322633003 & 0.4968837401810333\end{array}$

$\begin{array}{llll}0.6657598078712326 & 0.9981862417424736 & 0.9963482891271696\end{array}$

$\begin{array}{lllll}0.6671130549096560 & 0.3335565269548226 & 0.4949759688305448\end{array}$

$\begin{array}{llll}0.6656704909052777 & 0.3328352449526335 & 0.9954108739625269\end{array}$

$\begin{array}{lllll}0.6667344531316530 & 0.6665989208683527 & 0.4968837401810333\end{array}$

$\begin{array}{llll}0.6657598078712326 & 0.6675735661287732 & 0.9963482891271696\end{array}$

$\begin{array}{llll}0.1165719844665531 & 0.2167614555334509 & 0.0922517617861232\end{array}$

$\begin{array}{llll}0.1091381933323063 & 0.2241952466676977 & 0.6022985185037442\end{array}$

$\begin{array}{llll}0.1152061283507990 & 0.5576031501754031 & 0.1025792553508111\end{array}$

$\begin{array}{llll}0.1093525101224415 & 0.5546763410612243 & 0.6002476775482961\end{array}$

$\begin{array}{llll}0.1165719844665531 & 0.8998107599330822 & 0.0922517617861232\end{array}$

$\begin{array}{llll}0.1091381933323063 & 0.8849431776646099 & 0.6022985185037442\end{array}$

$\begin{array}{lllll}0.4335227030669131 & 0.2167614555334509 & 0.0922517617861232\end{array}$

$\begin{array}{lllll}0.4483902853353854 & 0.2241952466676977 & 0.6022985185037442\end{array}$

$\begin{array}{llll}0.4488355318071910 & 0.5643378096143863 & 0.1040579172704668\end{array}$ 
$\begin{array}{llll}0.4444644130289035 & 0.5555955720578183 & 0.6026797986964212\end{array}$ $\begin{array}{llll}0.4488355318071910 & 0.8844979311928043 & 0.1040579172704668\end{array}$ $\begin{array}{llll}0.4444644130289035 & 0.8888690499710918 & 0.6026797986964212\end{array}$ $\begin{array}{lllll}0.7757302828245969 & 0.2181273116492051 & 0.1025792553508111\end{array}$ $\begin{array}{llll}0.7786570919387756 & 0.2239809298775626 & 0.6002476775482961\end{array}$ $\begin{array}{lllll}0.7757302828245969 & 0.5576031501754031 & 0.1025792553508111\end{array}$ $\begin{array}{llll}0.7786570919387756 & 0.5546763410612243 & 0.6002476775482961\end{array}$ $\begin{array}{llll}0.7689956233856137 & 0.8844979311928043 & 0.1040579172704668\end{array}$ $\begin{array}{llll}0.7777378609421817 & 0.8888690499710918 & 0.6026797986964212\end{array}$ $\begin{array}{llll}0.2222223280000009 & 0.1111111120000032 & 0.3885690243415212\end{array}$ $\begin{array}{llll}0.2223010622973121 & 0.4451016064424493 & 0.3868173777427657\end{array}$ $\begin{array}{lllll}0.2253898119883146 & 0.4447313000336734 & 0.8862147372270357\end{array}$ $\begin{array}{lllll}0.2223010622973121 & 0.7771993668548589 & 0.3868173777427657\end{array}$ $\begin{array}{lllll}0.2253898119883146 & 0.7806584229546374 & 0.8862147372270357\end{array}$ $\begin{array}{lllll}0.5561340661451410 & 0.1110323777026920 & 0.3868173777427657\end{array}$ $\begin{array}{lllll}0.5526750100453626 & 0.1079436280116894 & 0.8862147372270357\end{array}$ $\begin{array}{llll}0.5561340661451410 & 0.4451016064424493 & 0.3868173777427657\end{array}$ $\begin{array}{lllll}0.5526750100453626 & 0.4447313000336734 & 0.8862147372270357\end{array}$ $\begin{array}{lllll}0.5555556420000016 & 0.7777777909999983 & 0.3877334888378385\end{array}$ $\begin{array}{llll}0.5555556420000016 & 0.7777777909999983 & 0.8858105447565663\end{array}$ $\begin{array}{llll}0.8882318565575460 & 0.1110323777026920 & 0.3868173777427657\end{array}$ 0.88860216296632190 .10794362801168940 .8862147372270357 $\begin{array}{lllll}0.8888890149999966 & 0.4444444479999987 & 0.3851056829434754\end{array}$ $\begin{array}{lllll}0.8888890149999966 & 0.4444444479999987 & 0.8877191690709765\end{array}$ $\begin{array}{llll}0.8882318565575460 & 0.7771993668548589 & 0.3868173777427657\end{array}$ $\begin{array}{lllll}0.8886021629663219 & 0.7806584229546374 & 0.8862147372270357\end{array}$ $\begin{array}{lllll}0.2222223280000009 & 0.1111109929999969 & 0.2825908208312882\end{array}$ $\begin{array}{lllll}0.2233340189419977 & 0.4501511279465475 & 0.2809682542104923\end{array}$ $\begin{array}{lllll}0.2301657422039014 & 0.4444204686755597 & 0.7803099400937015\end{array}$ $\begin{array}{llll}0.2233340189419977 & 0.7731825639954479 & 0.2809682542104923\end{array}$ $\begin{array}{llll}0.2301657422039014 & 0.7857449465283395 & 0.7803099400937015\end{array}$ $\begin{array}{llll}0.5601507500045528 & 0.1099993020580001 & 0.2809682542104923\end{array}$ $\begin{array}{llll}0.5475883674716613 & 0.1031675787960964 & 0.7803099400937015\end{array}$ $\begin{array}{lllll}0.5601507500045528 & 0.4501511279465475 & 0.2809682542104923\end{array}$ $\begin{array}{llll}0.5475883674716613 & 0.4444204686755597 & 0.7803099400937015\end{array}$ $\begin{array}{lllll}0.5555556420000016 & 0.7777776719999991 & 0.2814390179494026\end{array}$ $\begin{array}{lllll}0.5555556420000016 & 0.7777776719999991 & 0.7798223027189337\end{array}$ $\begin{array}{lllll}0.8831822160534486 & 0.1099993020580001 & 0.2809682542104923\end{array}$ $\begin{array}{lllll}0.8889128753244364 & 0.1031675787960964 & 0.7803099400937015\end{array}$ $\begin{array}{lllll}0.8888890149999966 & 0.4444443289999995 & 0.2790937703961589\end{array}$ $\begin{array}{lllll}0.8888890149999966 & 0.4444443289999995 & 0.7817931837818008\end{array}$ $\begin{array}{lllll}0.8831822160534486 & 0.7731825639954479 & 0.2809682542104923\end{array}$ $\begin{array}{llll}0.8889128753244364 & 0.7857449465283395 & 0.7803099400937015\end{array}$

\section{$\beta-\mathrm{NiOOH}$ structure(PBE0)-} 1.00000000000000
$8.7433362322307442 \quad 0.0000000248970828$
0.0000000000000000
$-4.3716691028010723 \quad 7.5719507213131436$
0.0000000000000000 
$\begin{array}{llll}0.0000000000000000 & 0.0000000000000000 & 9.0694469200061807\end{array}$

$\mathrm{Ni} \mathrm{O} \quad \mathrm{H}$

$\begin{array}{lll}18 & 36 & 18\end{array}$

Direct

$\begin{array}{llll}0.0000000000000000 & 0.0000000000000000 & 0.4970168159233737\end{array}$

$\begin{array}{llll}0.0000000000000000 & 0.0000000000000000 & 0.9970168159233737\end{array}$

$\begin{array}{lllll}0.0000000000000000 & 0.3333333429999996 & 0.4970168159233737\end{array}$

$\begin{array}{llll}0.0000000000000000 & 0.3333333429999996 & 0.9970168159233737\end{array}$

$\begin{array}{llll}0.0000000000000000 & 0.6666666870000029 & 0.4970168159233737\end{array}$

$\begin{array}{lllll}0.0000000000000000 & 0.6666666870000029 & 0.9970168159233737\end{array}$

$\begin{array}{lllll}0.3333333429999996 & 0.0000000000000000 & 0.4970168159233737\end{array}$

$\begin{array}{lllll}0.3333333429999996 & 0.0000000000000000 & 0.9970168159233737\end{array}$

$\begin{array}{lllll}0.3333333429999996 & 0.3333333429999996 & 0.4970168159233737\end{array}$

$\begin{array}{lllll}0.3333333429999996 & 0.3333333429999996 & 0.9970168159233737\end{array}$

$\begin{array}{lllll}0.3333333429999996 & 0.6666666870000029 & 0.4970168159233737\end{array}$

$\begin{array}{lllll}0.3333333429999996 & 0.6666666870000029 & 0.9970168159233737\end{array}$

$\begin{array}{lllll}0.6666666870000029 & 0.0000000000000000 & 0.4970168159233737\end{array}$

$\begin{array}{lllll}0.6666666870000029 & 0.0000000000000000 & 0.9970168159233737\end{array}$

$\begin{array}{llll}0.6666666870000029 & 0.3333333429999996 & 0.4970168159233737\end{array}$

$\begin{array}{llll}0.6666666870000029 & 0.3333333429999996 & 0.9970168159233737\end{array}$

$\begin{array}{llll}0.6666666870000029 & 0.6666666870000029 & 0.4970168159233737\end{array}$

$\begin{array}{llll}0.6666666870000029 & 0.6666666870000029 & 0.9970168159233737\end{array}$

$\begin{array}{llll}0.1111111120000032 & 0.2222223280000009 & 0.1021680634601339\end{array}$

$\begin{array}{llll}0.1111111120000032 & 0.2222223280000009 & 0.6021680864601322\end{array}$

$\begin{array}{llll}0.1111111120000032 & 0.5555556420000016 & 0.1021680634601339\end{array}$

$\begin{array}{llll}0.1111111120000032 & 0.5555556420000016 & 0.6021680864601322\end{array}$

$\begin{array}{llll}0.1111111120000032 & 0.8888890149999966 & 0.1021680634601339\end{array}$

$\begin{array}{llll}0.1111111120000032 & 0.8888890149999966 & 0.6021680864601322\end{array}$

$\begin{array}{lllll}0.4444444479999987 & 0.2222223280000009 & 0.1021680634601339\end{array}$

$\begin{array}{lllll}0.4444444479999987 & 0.2222223280000009 & 0.6021680864601322\end{array}$

$\begin{array}{llll}0.4444444479999987 & 0.5555556420000016 & 0.1021680634601339\end{array}$

$\begin{array}{lllll}0.4444444479999987 & 0.5555556420000016 & 0.6021680864601322\end{array}$

$\begin{array}{llllll}0.4444444479999987 & 0.8888890149999966 & 0.1021680634601339\end{array}$

$\begin{array}{llll}0.4444444479999987 & 0.8888890149999966 & 0.6021680864601322\end{array}$

$\begin{array}{llll}0.7777777909999983 & 0.2222223280000009 & 0.1021680634601339\end{array}$

$\begin{array}{lllll}0.7777777909999983 & 0.2222223280000009 & 0.6021680864601322\end{array}$

$\begin{array}{lllll}0.7777777909999983 & 0.5555556420000016 & 0.1021680634601339\end{array}$

$\begin{array}{lllll}0.7777777909999983 & 0.5555556420000016 & 0.6021680864601322\end{array}$

$\begin{array}{lllll}0.7777777909999983 & 0.8888890149999966 & 0.1021680634601339\end{array}$

$\begin{array}{llll}0.7777777909999983 & 0.8888890149999966 & 0.6021680864601322\end{array}$

$\begin{array}{llll}0.2222223280000009 & 0.1111111120000032 & 0.3865999328108103\end{array}$

$\begin{array}{llll}0.2222223280000009 & 0.1111111120000032 & 0.8865999038108114\end{array}$

$\begin{array}{lllll}0.2222223280000009 & 0.4444444479999987 & 0.3865999328108103\end{array}$

$\begin{array}{lllll}0.2222223280000009 & 0.4444444479999987 & 0.8865999038108114\end{array}$

$\begin{array}{llll}0.2222223280000009 & 0.7777777909999983 & 0.3865999328108103\end{array}$

$\begin{array}{llll}0.2222223280000009 & 0.7777777909999983 & 0.8865999038108114\end{array}$

$\begin{array}{llll}0.5555556420000016 & 0.1111111120000032 & 0.3865999328108103\end{array}$

$\begin{array}{lllll}0.5555556420000016 & 0.1111111120000032 & 0.8865999038108114\end{array}$

$\begin{array}{llll}0.5555556420000016 & 0.4444444479999987 & 0.3865999328108103\end{array}$ 
$\begin{array}{llll}0.5555556420000016 & 0.4444444479999987 & 0.8865999038108114\end{array}$ $\begin{array}{llll}0.5555556420000016 & 0.7777777909999983 & 0.3865999328108103\end{array}$ $\begin{array}{llll}0.5555556420000016 & 0.7777777909999983 & 0.8865999038108114\end{array}$ $\begin{array}{lllll}0.8888890149999966 & 0.1111111120000032 & 0.3865999328108103\end{array}$ $\begin{array}{llll}0.8888890149999966 & 0.1111111120000032 & 0.8865999038108114\end{array}$ $\begin{array}{lllll}0.8888890149999966 & 0.4444444479999987 & 0.3865999328108103\end{array}$ $\begin{array}{lllll}0.8888890149999966 & 0.4444444479999987 & 0.8865999038108114\end{array}$ $\begin{array}{llll}0.8888890149999966 & 0.7777777909999983 & 0.3865999328108103\end{array}$ $\begin{array}{llll}0.8888890149999966 & 0.7777777909999983 & 0.8865999038108114\end{array}$ $\begin{array}{llll}0.2222223280000009 & 0.1111109929999969 & 0.2792003088056916\end{array}$ $\begin{array}{lllll}0.2222223280000009 & 0.1111109929999969 & 0.7792003388056941\end{array}$ $\begin{array}{llll}0.2222223280000009 & 0.4444443289999995 & 0.2792003088056916\end{array}$ $\begin{array}{llll}0.2222223280000009 & 0.4444443289999995 & 0.7792003388056941\end{array}$ $\begin{array}{llll}0.2222223280000009 & 0.7777776719999991 & 0.2792003088056916\end{array}$ $\begin{array}{lllll}0.2222223280000009 & 0.7777776719999991 & 0.7792003388056941\end{array}$ $\begin{array}{lllll}0.5555556420000016 & 0.1111109929999969 & 0.2792003088056916\end{array}$ $\begin{array}{lllll}0.5555556420000016 & 0.1111109929999969 & 0.7792003388056941\end{array}$ $\begin{array}{llll}0.5555556420000016 & 0.4444443289999995 & 0.2792003088056916\end{array}$ $\begin{array}{lllll}0.5555556420000016 & 0.4444443289999995 & 0.7792003388056941\end{array}$ $\begin{array}{llll}0.5555556420000016 & 0.7777776719999991 & 0.2792003088056916\end{array}$ $\begin{array}{lllll}0.5555556420000016 & 0.7777776719999991 & 0.7792003388056941\end{array}$ $\begin{array}{llll}0.8888890149999966 & 0.1111109929999969 & 0.2792003088056916\end{array}$ $\begin{array}{lllll}0.8888890149999966 & 0.1111109929999969 & 0.7792003388056941\end{array}$ $\begin{array}{lllll}0.8888890149999966 & 0.4444443289999995 & 0.2792003088056916\end{array}$ $\begin{array}{lllll}0.8888890149999966 & 0.4444443289999995 & 0.7792003388056941\end{array}$ $\begin{array}{lllll}0.8888890149999966 & 0.7777776719999991 & 0.2792003088056916\end{array}$ $\begin{array}{llll}0.8888890149999966 & 0.7777776719999991 & 0.7792003388056941\end{array}$

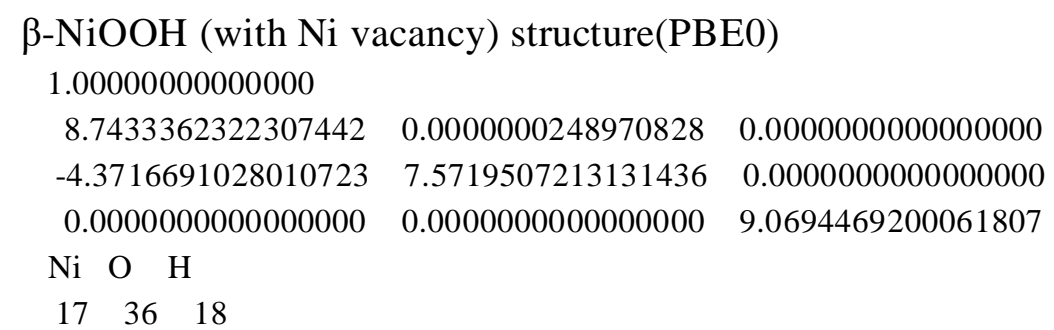

Direct

$\begin{array}{llll}0.0010989514291282 & 0.9997723205661728 & 0.4961831218233570\end{array}$ $\begin{array}{llll}0.0026061941919693 & 0.9989524849586289 & 0.9939605742072857\end{array}$ $\begin{array}{llll}0.0000000000000000 & 0.3333333429999996 & 0.4948131798054831\end{array}$ $\begin{array}{lllll}0.0000000000000000 & 0.3333333429999996 & 0.9990100530802124\end{array}$ $\begin{array}{lllll}0.0010989514291282 & 0.6679933178629582 & 0.4961831218233570\end{array}$ $\begin{array}{lllll}0.0026061941919693 & 0.6703203962333646 & 0.9939605742072857\end{array}$ $\begin{array}{llll}0.3333333429999996 & 0.0000000000000000 & 0.9990230669060836\end{array}$ $\begin{array}{lllll}0.3335610224338268 & 0.3322343915708714 & 0.4961831218233570\end{array}$ $\begin{array}{llll}0.3343808580413778 & 0.3307271488080303 & 0.9939605742072857\end{array}$ $\begin{array}{lllll}0.3335610224338268 & 0.6679933178629582 & 0.4961831218233570\end{array}$ $\begin{array}{llll}0.3343808580413778 & 0.6703203962333646 & 0.9939605742072857\end{array}$ $\begin{array}{llll}0.6653400561370475 & 0.9997723205661728 & 0.4961831218233570\end{array}$ 
$\begin{array}{llll}0.6630129777666411 & 0.9989524849586289 & 0.9939605742072857\end{array}$ $\begin{array}{lllll}0.6653400561370475 & 0.3322343915708714 & 0.4961831218233570\end{array}$ $\begin{array}{llll}0.6630129777666411 & 0.3307271488080303 & 0.9939605742072857\end{array}$ $\begin{array}{llll}0.6666666870000029 & 0.6666666870000029 & 0.4972226980085068\end{array}$ $\begin{array}{llll}0.6666666870000029 & 0.6666666870000029 & 0.0087273150747507\end{array}$ $\begin{array}{lllll}0.1220744497968127 & 0.2112589902031914 & 0.0969124307323312\end{array}$ $\begin{array}{llll}0.1169269040422662 & 0.2164065359577378 & 0.5994410147952536\end{array}$ $\begin{array}{llll}0.1220744497968127 & 0.5774823175936206 & 0.0969124307323312\end{array}$ $\begin{array}{lllll}0.1169269040422662 & 0.5671872260845277 & 0.5994410147952536\end{array}$ $\begin{array}{lllll}0.0993900870997777 & 0.8830285025498910 & 0.1012559147132208\end{array}$ $\begin{array}{llll}0.0678289503077139 & 0.8672479341538519 & 0.6130011251530618\end{array}$ $\begin{array}{lllll}0.4503049604501044 & 0.2339433529002264 & 0.1012559147132208\end{array}$ $\begin{array}{llll}0.4660855288461434 & 0.2655044896922902 & 0.6130011251530618\end{array}$ $\begin{array}{llll}0.4267644485776430 & 0.5467156422888308 & 0.1069083244218518\end{array}$ $\begin{array}{llll}0.4274888631183984 & 0.5470778495592015 & 0.5968572964452434\end{array}$ $\begin{array}{lllll}0.4503049604501044 & 0.8830285025498910 & 0.1012559147132208\end{array}$ $\begin{array}{lllll}0.4660855288461434 & 0.8672479341538519 & 0.6130011251530618\end{array}$ $\begin{array}{llll}0.7558511154063794 & 0.2112589902031914 & 0.0969124307323312\end{array}$ $\begin{array}{llll}0.7661462069154723 & 0.2164065359577378 & 0.5994410147952536\end{array}$ $\begin{array}{llll}0.7866177907111691 & 0.5467156422888308 & 0.1069083244218518\end{array}$ $\begin{array}{llll}0.7862555834407985 & 0.5470778495592015 & 0.5968572964452434\end{array}$ $\begin{array}{llll}0.7866177907111691 & 0.9065690144223524 & 0.1069083244218518\end{array}$ $\begin{array}{llll}0.7862555834407985 & 0.9058445998816040 & 0.5968572964452434\end{array}$ $\begin{array}{lllll}0.2184524979924731 & 0.1148809420075310 & 0.3939590800432455\end{array}$ $\begin{array}{llll}0.2229649959107078 & 0.1103684440892962 & 0.8793039099490514\end{array}$ $\begin{array}{lllll}0.2315281372943119 & 0.4490973526471578 & 0.3826721293896682\end{array}$ $\begin{array}{lllll}0.2319405797735214 & 0.4493035738867590 & 0.8854896425926313\end{array}$ $\begin{array}{lllll}0.2184524979924731 & 0.7702381309849500 & 0.3939590800432455\end{array}$ $\begin{array}{llll}0.2229649959107078 & 0.7792631268214265 & 0.8793039099490514\end{array}$ $\begin{array}{lllll}0.5630953020150500 & 0.1148809420075310 & 0.3939590800432455\end{array}$ $\begin{array}{llll}0.5540703061785734 & 0.1103684440892962 & 0.8793039099490514\end{array}$ $\begin{array}{lllll}0.5500447122038068 & 0.4334225884076019 & 0.3849347230959452\end{array}$ $\begin{array}{lllll}0.5509944259920516 & 0.4353220159840916 & 0.8884491406689534\end{array}$ $\begin{array}{lllll}0.5500447122038068 & 0.7832887207961861 & 0.3849347230959452\end{array}$ $\begin{array}{llll}0.5509944259920516 & 0.7823390070079483 & 0.8884491406689534\end{array}$ $\begin{array}{lllll}0.8842361103528376 & 0.1018053027056922 & 0.3826721293896682\end{array}$ $\begin{array}{lllll}0.8840298891132363 & 0.1013928602264826 & 0.8854896425926313\end{array}$ $\begin{array}{lllll}0.8842361103528376 & 0.4490973526471578 & 0.3826721293896682\end{array}$ $\begin{array}{lllll}0.8840298891132363 & 0.4493035738867590 & 0.8854896425926313\end{array}$ $\begin{array}{lllll}0.8999108745923934 & 0.7832887207961861 & 0.3849347230959452\end{array}$ $\begin{array}{llll}0.8980114470159037 & 0.7823390070079483 & 0.8884491406689534\end{array}$ $\begin{array}{lllll}0.1973381030807815 & 0.1359952179192163 & 0.2918702120573258\end{array}$ $\begin{array}{lllll}0.2210335030569865 & 0.1122998179430112 & 0.7720613783611157\end{array}$ $\begin{array}{llll}0.2647411896469407 & 0.4657037598234695 & 0.2779414577668078\end{array}$ $\begin{array}{llll}0.2456525406510295 & 0.4561594353255245 & 0.7787144115522793\end{array}$ $\begin{array}{lllll}0.1973381030807815 & 0.7280092221615604 & 0.2918702120573258\end{array}$ $\begin{array}{lllll}0.2210335030569865 & 0.7754000221139634 & 0.7720613783611157\end{array}$ $\begin{array}{lllll}0.6053240918384404 & 0.1359952179192163 & 0.2918702120573258\end{array}$ $\begin{array}{llll}0.5579332918860374 & 0.1122998179430112 & 0.7720613783611157\end{array}$ 
$\begin{array}{llll}0.5368525641733655 & 0.4070381733467485 & 0.2791331831890815\end{array}$ $\begin{array}{llll}0.5351169387990780 & 0.4035669225981593 & 0.7821136547455509\end{array}$ $\begin{array}{llll}0.5368525641733655 & 0.7964807498266353 & 0.2791331831890815\end{array}$ $\begin{array}{llll}0.5351169387990780 & 0.7982163752009228 & 0.7821136547455509\end{array}$ $\begin{array}{llll}0.8676295841765267 & 0.0685921313530571 & 0.2779414577668078\end{array}$ $\begin{array}{llll}0.8771739086744788 & 0.0876807803489683 & 0.7787144115522793\end{array}$ $\begin{array}{llll}0.8676295841765267 & 0.4657037598234695 & 0.2779414577668078\end{array}$ $\begin{array}{llll}0.8771739086744788 & 0.4561594353255245 & 0.7787144115522793\end{array}$ $\begin{array}{llll}0.9262951706532405 & 0.7964807498266353 & 0.2791331831890815\end{array}$ $\begin{array}{llll}0.9297664214018369 & 0.7982163752009228 & 0.7821136547455509\end{array}$

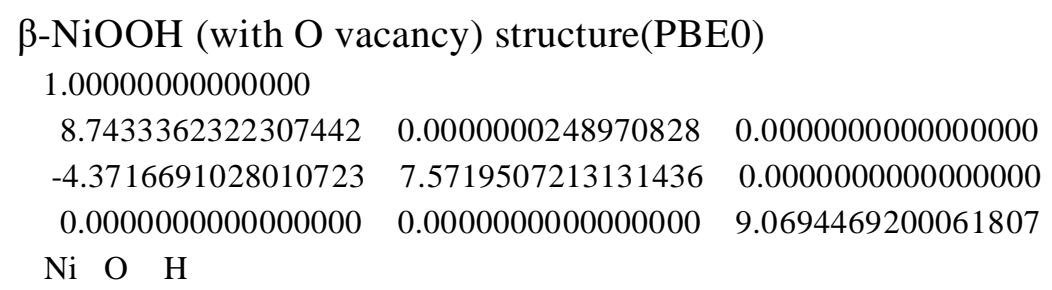
$\begin{array}{lll}18 & 35 & 18\end{array}$

Direct

$\begin{array}{llll}0.0090278949487583 & 0.0045139474743792 & 0.4912154165015465\end{array}$ $\begin{array}{llll}0.0010092083023494 & 0.0005046041511747 & 0.9953888059543630\end{array}$ $\begin{array}{llll}0.9980441814165673 & 0.3294217058331128 & 0.5053045919137560\end{array}$ $\begin{array}{lllll}0.0001321724540233 & 0.3335976879080604 & 0.9987428171065318\end{array}$ $\begin{array}{lllll}0.9980441814165673 & 0.6686225055834356 & 0.5053045919137560\end{array}$ $\begin{array}{lllll}0.0001321724540233 & 0.6665345145459796 & 0.9987428171065318\end{array}$ $\begin{array}{llll}0.3344599436623739 & 0.0022532013247485 & 0.4957754099119072\end{array}$ $\begin{array}{llll}0.3333544197441540 & 0.0000421534883301 & 0.9937070572075797\end{array}$ $\begin{array}{llll}0.3344599436623739 & 0.3322067423376254 & 0.4957754099119072\end{array}$ $\begin{array}{llll}0.3333544197441540 & 0.3333122662558452 & 0.9937070572075797\end{array}$ $\begin{array}{llll}0.3372449801668864 & 0.6686225055834356 & 0.5053045919137560\end{array}$ $\begin{array}{llll}0.3330689980919388 & 0.6665345145459796 & 0.9987428171065318\end{array}$ $\begin{array}{llll}0.6621527395256095 & 0.0045139474743792 & 0.4912154165015465\end{array}$ $\begin{array}{llll}0.6661620828488282 & 0.0005046041511747 & 0.9953888059543630\end{array}$ $\begin{array}{lllll}0.6644134856752544 & 0.3322067423376254 & 0.4957754099119072\end{array}$ $\begin{array}{llll}0.6666245335116798 & 0.3333122662558452 & 0.9937070572075797\end{array}$ $\begin{array}{llll}0.6621527395256095 & 0.6576387920512445 & 0.4912154165015465\end{array}$ $\begin{array}{llll}0.6661620828488282 & 0.6656574786976535 & 0.9953888059543630\end{array}$ $\begin{array}{lllll}0.1132121589118498 & 0.2231675799642829 & 0.1004838558292249\end{array}$ $\begin{array}{llll}0.1011604869727236 & 0.2039484925848853 & 0.6044695617736124\end{array}$ $\begin{array}{llll}0.1111111120000032 & 0.5555556420000016 & 0.1032515436682075\end{array}$ $\begin{array}{llll}0.1111111120000032 & 0.5555556420000016 & 0.6170966411588950\end{array}$ $\begin{array}{llll}0.1132121589118498 & 0.8900448099475682 & 0.1004838558292249\end{array}$ $\begin{array}{llll}0.1011604869727236 & 0.8972122253878183 & 0.6044695617736124\end{array}$ $\begin{array}{llll}0.4444444479999987 & 0.2222223280000009 & 0.0964029449079931\end{array}$ $\begin{array}{lllll}0.4444444479999987 & 0.2222223280000009 & 0.5960835565845315\end{array}$ $\begin{array}{lllll}0.4434991960357166 & 0.5534545950881551 & 0.1004838558292249\end{array}$ $\begin{array}{lllll}0.4627182834151142 & 0.5655062670272741 & 0.6044695617736124\end{array}$ $\begin{array}{llll}0.4434991960357166 & 0.8900448099475682 & 0.1004838558292249\end{array}$ 
$\begin{array}{llll}0.4627182834151142 & 0.8972122253878183 & 0.6044695617736124\end{array}$ $\begin{array}{llll}0.7766219960524268 & 0.2231675799642829 & 0.1004838558292249\end{array}$ $\begin{array}{llll}0.7694545806121837 & 0.2039484925848853 & 0.6044695617736124\end{array}$ $\begin{array}{llll}0.7766219960524268 & 0.5534545950881551 & 0.1004838558292249\end{array}$ $\begin{array}{llll}0.7694545806121837 & 0.5655062670272741 & 0.6044695617736124\end{array}$ $\begin{array}{lllll}0.7777777909999983 & 0.8888890149999966 & 0.1022588242477696\end{array}$ $\begin{array}{llll}0.2228106032530661 & 0.1114052496265288 & 0.3752238534518639\end{array}$ $\begin{array}{lllll}0.2217868265630614 & 0.1108933612815406 & 0.8839902478815418\end{array}$ $\begin{array}{lllll}0.2213915960661197 & 0.4452751799338728 & 0.3955014076029499\end{array}$ $\begin{array}{lllll}0.2215322652988050 & 0.4451345107012017 & 0.8862184561973407\end{array}$ $\begin{array}{llll}0.2213915960661197 & 0.7761163271322431 & 0.3955014076029499\end{array}$ $\begin{array}{llll}0.2215322652988050 & 0.7763976655975782 & 0.8862184561973407\end{array}$ $\begin{array}{llll}0.5552615043734690 & 0.1114052496265288 & 0.3752238534518639\end{array}$ $\begin{array}{llll}0.5557733927184643 & 0.1108933612815406 & 0.8839902478815418\end{array}$ $\begin{array}{lllll}0.5552615043734690 & 0.4438561727469335 & 0.3752238534518639\end{array}$ $\begin{array}{lllll}0.5557733927184643 & 0.4448799494369382 & 0.8839902478815418\end{array}$ $\begin{array}{llll}0.5684946392131565 & 0.7842472896065829 & 0.3918554140146924\end{array}$ $\begin{array}{lllll}0.5532419125073105 & 0.7766209262536421 & 0.8852533800920526\end{array}$ $\begin{array}{lllll}0.8824195163934121 & 0.0981721147868484 & 0.3918554140146924\end{array}$ $\begin{array}{llll}0.8900458797463529 & 0.1134248414926944 & 0.8852533800920526\end{array}$ $\begin{array}{llll}0.8905504788677518 & 0.4452751799338728 & 0.3955014076029499\end{array}$ $\begin{array}{lllll}0.8902691404024168 & 0.4451345107012017 & 0.8862184561973407\end{array}$ $\begin{array}{llll}0.8824195163934121 & 0.7842472896065829 & 0.3918554140146924\end{array}$ $\begin{array}{llll}0.8900458797463529 & 0.7766209262536421 & 0.8852533800920526\end{array}$ $\begin{array}{llll}0.2203942588361443 & 0.1101969584180793 & 0.2683809006226170\end{array}$ $\begin{array}{lllll}0.2157193714903514 & 0.1078595147451651 & 0.7765158673536803\end{array}$ $\begin{array}{llll}0.2205358529905865 & 0.4461308040094139 & 0.2883116730706519\end{array}$ $\begin{array}{lllll}0.2176240406557000 & 0.4490426163443004 & 0.7792024455887017\end{array}$ $\begin{array}{lllll}0.2205358529905865 & 0.7744047219811705 & 0.2883116730706519\end{array}$ $\begin{array}{lllll}0.2176240406557000 & 0.7685810973113831 & 0.7792024455887017\end{array}$ $\begin{array}{llll}0.5564696765819193 & 0.1101969584180793 & 0.2683809006226170\end{array}$ $\begin{array}{llll}0.5588071202548264 & 0.1078595147451651 & 0.7765158673536803\end{array}$ $\begin{array}{lllll}0.5564696765819193 & 0.4462723981638561 & 0.2683809006226170\end{array}$ $\begin{array}{lllll}0.5588071202548264 & 0.4509472855096419 & 0.7765158673536803\end{array}$ $\begin{array}{lllll}0.5798620637344456 & 0.7899308828672176 & 0.2847066807165604\end{array}$ $\begin{array}{lllll}0.5355088551899669 & 0.7677542785949782 & 0.7789556719302269\end{array}$ $\begin{array}{llll}0.8767358041327782 & 0.0868045712655530 & 0.2847066807165604\end{array}$ $\begin{array}{llll}0.8989124084050175 & 0.1311577798100245 & 0.7789556719302269\end{array}$ $\begin{array}{lllll}0.8922619650188253 & 0.4461308040094139 & 0.2883116730706519\end{array}$ $\begin{array}{lllll}0.8980855896886126 & 0.4490426163443004 & 0.7792024455887017\end{array}$ $\begin{array}{lllll}0.8767358041327782 & 0.7899308828672176 & 0.2847066807165604\end{array}$ $\begin{array}{llll}0.8989124084050175 & 0.7677542785949782 & 0.7789556719302269\end{array}$

\author{
$\beta-\mathrm{NiOOH}$ (with $\mathrm{H}$ vacancy) structure(PBE0) \\ 1.00000000000000

$\begin{array}{rrr}8.7433362322307442 & 0.0000000248970828 & 0.0000000000000000 \\ -4.3716691028010723 & 7.5719507213131436 & 0.0000000000000000 \\ 0.000000000000000 & 0.0000000000000000 & 9.0694469200061807\end{array}$


$\mathrm{Ni} \quad \mathrm{O} \quad \mathrm{H}$

$\begin{array}{lll}18 & 36 & 17\end{array}$

Direct

$\begin{array}{llll}0.0011916988481957 & 0.9988083011518043 & 0.4991433871196378\end{array}$

$\begin{array}{llll}0.9974965343899242 & 0.0025034656100758 & 0.9935796362988540\end{array}$

$\begin{array}{llll}0.0011916988481957 & 0.3357167406964123 & 0.4991433871196378\end{array}$

$\begin{array}{llll}0.9974965343899242 & 0.3283264117798339 & 0.9935796362988540\end{array}$

$\begin{array}{lllll}0.0022413479928005 & 0.6677873609964067 & 0.4890992602837017\end{array}$

$\begin{array}{llll}0.0073400858823121 & 0.6703367299411696 & 0.9915131022493995\end{array}$

$\begin{array}{llll}0.3322126690035958 & 0.9977586520071995 & 0.4890992602837017\end{array}$

$\begin{array}{llll}0.3296633000588329 & 0.9926599141176879 & 0.9915131022493995\end{array}$

$\begin{array}{lllll}0.3450169769346871 & 0.3391751599673398 & 0.4971235136535910\end{array}$

$\begin{array}{llll}0.3318316275971327 & 0.3325824852985662 & 0.0128548483962447\end{array}$

$\begin{array}{llll}0.3322126690035958 & 0.6677873609964067 & 0.4890992602837017\end{array}$

$\begin{array}{llll}0.3296633000588329 & 0.6703367299411696 & 0.9915131022493995\end{array}$

$\begin{array}{llll}0.6642832893035902 & 0.9988083011518043 & 0.4991433871196378\end{array}$

$\begin{array}{llll}0.6716736182201757 & 0.0025034656100758 & 0.9935796362988540\end{array}$

$\begin{array}{llll}0.6608248700326627 & 0.3391751599673398 & 0.4971235136535910\end{array}$

$\begin{array}{llll}0.6674175447014363 & 0.3325824852985662 & 0.0128548483962447\end{array}$

$\begin{array}{llll}0.6608248700326627 & 0.6549830530653153 & 0.4971235136535910\end{array}$

$\begin{array}{llll}0.6674175447014363 & 0.6681684024028698 & 0.0128548483962447\end{array}$

$\begin{array}{llll}0.0919426767823950 & 0.2126381103912038 & 0.0984230292425963\end{array}$

$\begin{array}{lllll}0.1366105000569107 & 0.2349720220284510 & 0.6036886763227045\end{array}$

$\begin{array}{llll}0.1210846212367827 & 0.5755026604735534 & 0.0941155670166864\end{array}$

$\begin{array}{llll}0.1187012487701296 & 0.5707359155402543 & 0.5919345404957710\end{array}$

$\begin{array}{llll}0.1210846212367827 & 0.8789155057632243 & 0.0941155670166864\end{array}$

$\begin{array}{llll}0.1187012487701296 & 0.8812988782298703 & 0.5919345404957710\end{array}$

$\begin{array}{lllll}0.4245925459639039 & 0.1825185239277971 & 0.1153962285984917\end{array}$

$\begin{array}{lllll}0.4535544578909594 & 0.2404423477819364 & 0.6020606471023981\end{array}$

$\begin{array}{llll}0.4245925459639039 & 0.5754075440360964 & 0.1153962285984917\end{array}$

$\begin{array}{llll}0.4535544578909594 & 0.5464456321090339 & 0.6020606471023981\end{array}$

$\begin{array}{llll}0.4244974295264470 & 0.8789155057632243 & 0.0941155670166864\end{array}$

$\begin{array}{llll}0.4292641744597390 & 0.8812988782298703 & 0.5919345404957710\end{array}$

$\begin{array}{llll}0.7873620086087953 & 0.2126381103912038 & 0.0984230292425963\end{array}$

$\begin{array}{lllll}0.7650280969715411 & 0.2349720220284510 & 0.6036886763227045\end{array}$

$\begin{array}{lllll}0.8174815950722021 & 0.5754075440360964 & 0.1153962285984917\end{array}$

$\begin{array}{llll}0.7595577712180628 & 0.5464456321090339 & 0.6020606471023981\end{array}$

$\begin{array}{llll}0.7873620086087953 & 0.9080574502176049 & 0.0984230292425963\end{array}$

$\begin{array}{lllll}0.7650280969715411 & 0.8633896269430892 & 0.6036886763227045\end{array}$

$\begin{array}{llll}0.2392512066834485 & 0.1244861017806045 & 0.3869426887380172\end{array}$ $\begin{array}{lllll}0.2222729542287283 & 0.1043429402930798 & 0.8903847920688577\end{array}$ $\begin{array}{lllll}0.2392512066834485 & 0.4480983369028380 & 0.3869426887380172\end{array}$ $\begin{array}{llll}0.2222729542287283 & 0.4512632459356425 & 0.8903847920688577\end{array}$ $\begin{array}{llll}0.2222223280000009 & 0.7777777909999983 & 0.3661174546708708\end{array}$ $\begin{array}{llll}0.2222223280000009 & 0.7777777909999983 & 0.8768396457504579\end{array}$ $\begin{array}{llll}0.5519017530971553 & 0.1244861017806045 & 0.3869426887380172\end{array}$ $\begin{array}{llll}0.5487368440643579 & 0.1043429402930798 & 0.8903847920688577\end{array}$ $\begin{array}{lllll}0.5555556420000016 & 0.4444444479999987 & 0.3906256187221118\end{array}$ $\begin{array}{llll}0.5555556420000016 & 0.4444444479999987 & 0.9040933545580643\end{array}$ 
$\begin{array}{llll}0.5519017530971553 & 0.7607489123165507 & 0.3869426887380172\end{array}$ $\begin{array}{lllll}0.5487368440643579 & 0.7777271647712709 & 0.8903847920688577\end{array}$ $\begin{array}{lllll}0.8888890149999966 & 0.1111111120000032 & 0.3937226419298838\end{array}$ $\begin{array}{lllll}0.8888890149999966 & 0.1111111120000032 & 0.8677574000251340\end{array}$ $\begin{array}{llll}0.8755140252193954 & 0.4480983369028380 & 0.3869426887380172\end{array}$ $\begin{array}{lllll}0.8956571867069201 & 0.4512632459356425 & 0.8903847920688577\end{array}$ $\begin{array}{lllll}0.8755140252193954 & 0.7607489123165507 & 0.3869426887380172\end{array}$ $\begin{array}{llll}0.8956571867069201 & 0.7777271647712709 & 0.8903847920688577\end{array}$ $\begin{array}{llll}0.2710930094854120 & 0.1380599457407570 & 0.2815975117267513\end{array}$ $\begin{array}{lllll}0.2226967254815762 & 0.1103941427736004 & 0.7831560882028583\end{array}$ $\begin{array}{llll}0.2710930094854120 & 0.4663660577446649 & 0.2815975117267513\end{array}$ $\begin{array}{llll}0.2226967254815762 & 0.4456355767079572 & 0.7831560882028583\end{array}$ $\begin{array}{llll}0.2222223280000009 & 0.7777776719999991 & 0.2590884603844614\end{array}$ $\begin{array}{lllll}0.2222223280000009 & 0.7777776719999991 & 0.7685809744745669\end{array}$ $\begin{array}{llll}0.5336339132553363 & 0.1380599457407570 & 0.2815975117267513\end{array}$ $\begin{array}{lllll}0.5543643942920440 & 0.1103941427736004 & 0.7831560882028583\end{array}$ $\begin{array}{lllll}0.5555556420000016 & 0.4444443289999995 & 0.7972398484950531\end{array}$ $\begin{array}{llll}0.5336339132553363 & 0.7289069905145809 & 0.2815975117267513\end{array}$ $\begin{array}{lllll}0.5543643942920440 & 0.7773032745184310 & 0.7831560882028583\end{array}$ $\begin{array}{lllll}0.8888890149999966 & 0.1111109929999969 & 0.2857945610352459\end{array}$ $\begin{array}{lllll}0.8888890149999966 & 0.1111109929999969 & 0.7613883303894653\end{array}$ $\begin{array}{lllll}0.8619400622592366 & 0.4663660577446649 & 0.2815975117267513\end{array}$ $\begin{array}{lllll}0.8896058652263932 & 0.4456355767079572 & 0.7831560882028583\end{array}$ $\begin{array}{llll}0.8619400622592366 & 0.7289069905145809 & 0.2815975117267513\end{array}$ $\begin{array}{llll}0.8896058652263932 & 0.7773032745184310 & 0.7831560882028583\end{array}$

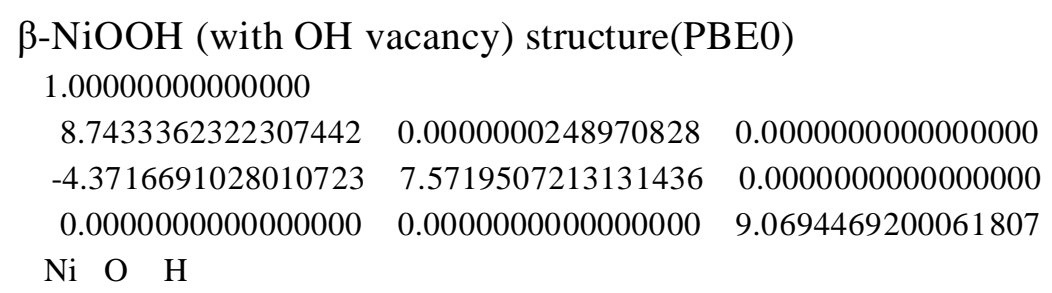
$\begin{array}{lll}18 & 35 & 17\end{array}$

Direct

0.00023112011216850 .00011556005609490 .5035167795013962 $\begin{array}{llll}0.9935077729737429 & 0.9967538864868715 & 0.9968671356201710\end{array}$ $\begin{array}{lllll}0.9961449847772315 & 0.3371883582227682 & 0.4976877505424895\end{array}$ $\begin{array}{lllll}0.9999994641713101 & 0.3333338788286895 & 0.0047390290666911\end{array}$ $\begin{array}{llll}0.9961449847772315 & 0.6589566565545013 & 0.4976877505424895\end{array}$ $\begin{array}{lllll}0.9999994641713101 & 0.6666656153426374 & 0.0047390290666911\end{array}$ $\begin{array}{llll}0.3332177829439047 & 0.0001155600560949 & 0.5035167795013962\end{array}$ $\begin{array}{llll}0.3365794565131282 & 0.9967538864868715 & 0.9968671356201710\end{array}$ $\begin{array}{lllll}0.3332177829439047 & 0.3331022228878311 & 0.5035167795013962\end{array}$ $\begin{array}{llll}0.3365794565131282 & 0.3398255700262567 & 0.9968671356201710\end{array}$ $\begin{array}{lllll}0.3367776921465335 & 0.6683888615732556 & 0.4935420733672089\end{array}$ $\begin{array}{llll}0.3387931988974913 & 0.6693966149487593 & 0.9910624513472044\end{array}$ $\begin{array}{llll}0.6649445124267501 & 0.9965556508534661 & 0.4935420733672089\end{array}$ $\begin{array}{llll}0.6639367590512464 & 0.9945401441025083 & 0.9910624513472044\end{array}$ 
$\begin{array}{llll}0.6743767174455044 & 0.3371883582227682 & 0.4976877505424895\end{array}$ $\begin{array}{lllll}0.6666677586573684 & 0.3333338788286895 & 0.0047390290666911\end{array}$ $\begin{array}{lllll}0.6649445124267501 & 0.6683888615732556 & 0.4935420733672089\end{array}$ $\begin{array}{llll}0.6639367590512464 & 0.6693966149487593 & 0.9910624513472044\end{array}$ $\begin{array}{llll}0.1201862917741892 & 0.2131471482258149 & 0.0883831627132281\end{array}$ $\begin{array}{lllll}0.0952864421317230 & 0.2380469978682811 & 0.6100200535513309\end{array}$ $\begin{array}{llll}0.1309993306467661 & 0.5654997513233795 & 0.1141993542988899\end{array}$ $\begin{array}{llll}0.0931738515375784 & 0.5465870117687786 & 0.6019763091596104\end{array}$ $\begin{array}{lllll}0.1201862917741892 & 0.9070393745483756 & 0.0883831627132281\end{array}$ $\begin{array}{lllll}0.0952864421317230 & 0.8572396752634290 & 0.6100200535513309\end{array}$ $\begin{array}{lllll}0.4262940884516198 & 0.2131471482258149 & 0.0883831627132281\end{array}$ $\begin{array}{lllll}0.4760937877365663 & 0.2380469978682811 & 0.6100200535513309\end{array}$ $\begin{array}{llll}0.4539164910046694 & 0.5744997280093429 & 0.0939530949704803\end{array}$ $\begin{array}{llll}0.4526251098562710 & 0.5719169657125462 & 0.5961801390001042\end{array}$ $\begin{array}{llll}0.4539164910046694 & 0.8794169719953260 & 0.0939530949704803\end{array}$ $\begin{array}{llll}0.4526251098562710 & 0.8807083531437243 & 0.5961801390001042\end{array}$ $\begin{array}{lllll}0.7678336816766205 & 0.2023341093532380 & 0.1141993542988899\end{array}$ $\begin{array}{lllll}0.7867464212312143 & 0.2401595884624257 & 0.6019763091596104\end{array}$ $\begin{array}{llll}0.7678336816766205 & 0.5654997513233795 & 0.1141993542988899\end{array}$ $\begin{array}{lllll}0.7867464212312143 & 0.5465870117687786 & 0.6019763091596104\end{array}$ $\begin{array}{llll}0.7588337049906571 & 0.8794169719953260 & 0.0939530949704803\end{array}$ $\begin{array}{llll}0.7614164672874537 & 0.8807083531437243 & 0.5961801390001042\end{array}$ $\begin{array}{llll}0.2222223280000009 & 0.1111111120000032 & 0.3921569440006962\end{array}$ $\begin{array}{llll}0.2097511684362559 & 0.4466015431921733 & 0.3889270896400561\end{array}$ $\begin{array}{lllll}0.2286523079871401 & 0.4511763546186245 & 0.8870949461898903\end{array}$ $\begin{array}{lllll}0.2097511684362559 & 0.7631495362440717 & 0.3889270896400561\end{array}$ $\begin{array}{lllll}0.2286523079871401 & 0.7774758643685118 & 0.8870949461898903\end{array}$ $\begin{array}{lllll}0.5701838967559283 & 0.1235822715637482 & 0.3889270896400561\end{array}$ $\begin{array}{llll}0.5558575686314882 & 0.1046811320128640 & 0.8870949461898903\end{array}$ $\begin{array}{llll}0.5701838967559283 & 0.4466015431921733 & 0.3889270896400561\end{array}$ $\begin{array}{llll}0.5558575686314882 & 0.4511763546186245 & 0.8870949461898903\end{array}$ $\begin{array}{lllll}0.5555556420000016 & 0.7777777909999983 & 0.3692027270211682\end{array}$ $\begin{array}{lllll}0.5555556420000016 & 0.7777777909999983 & 0.8730925554411471\end{array}$ $\begin{array}{lllll}0.8867319198078221 & 0.1235822715637482 & 0.3889270896400561\end{array}$ $\begin{array}{lllll}0.8821571083813708 & 0.1046811320128640 & 0.8870949461898903\end{array}$ $\begin{array}{lllll}0.8888890149999966 & 0.4444444479999987 & 0.3846626123992536\end{array}$ $\begin{array}{lllll}0.8888890149999966 & 0.4444444479999987 & 0.8954112477187692\end{array}$ $\begin{array}{lllll}0.8867319198078221 & 0.7631495362440717 & 0.3889270896400561\end{array}$ $\begin{array}{llll}0.8821571083813708 & 0.7774758643685118 & 0.8870949461898903\end{array}$ $\begin{array}{lllll}0.2222223280000009 & 0.1111109929999969 & 0.2853371466994332\end{array}$ $\begin{array}{lllll}0.1953436778552557 & 0.4618502355823679 & 0.2836127218791162\end{array}$ $\begin{array}{lllll}0.2293494908080831 & 0.4492129226598536 & 0.7795826762163003\end{array}$ $\begin{array}{llll}0.1953436778552557 & 0.7334931152728998 & 0.2836127218791162\end{array}$ $\begin{array}{llll}0.2293494908080831 & 0.7801362411482202 & 0.7795826762163003\end{array}$ $\begin{array}{lllll}0.5998401987271009 & 0.1379896431447420 & 0.2836127218791162\end{array}$ $\begin{array}{lllll}0.5531970728517805 & 0.1039838301919147 & 0.7795826762163003\end{array}$ $\begin{array}{lllll}0.5998401987271009 & 0.4618502355823679 & 0.2836127218791162\end{array}$ $\begin{array}{lllll}0.5531970728517805 & 0.4492129226598536 & 0.7795826762163003\end{array}$ $\begin{array}{llll}0.5555556420000016 & 0.7777776719999991 & 0.2624267748448403\end{array}$ 
$\begin{array}{llll}0.5555556420000016 & 0.7777776719999991 & 0.7647186194327986\end{array}$ $\begin{array}{llll}0.8714831084176282 & 0.1379896431447420 & 0.2836127218791162\end{array}$ $\begin{array}{lllll}0.8841204213401426 & 0.1039838301919147 & 0.7795826762163003\end{array}$ $\begin{array}{llll}0.8888890149999966 & 0.4444443289999995 & 0.2770462824379791\end{array}$ $\begin{array}{llll}0.8888890149999966 & 0.4444443289999995 & 0.7881906384190955\end{array}$ $\begin{array}{llll}0.8714831084176282 & 0.7334931152728998 & 0.2836127218791162\end{array}$ $\begin{array}{llll}0.8841204213401426 & 0.7801362411482202 & 0.7795826762163003\end{array}$

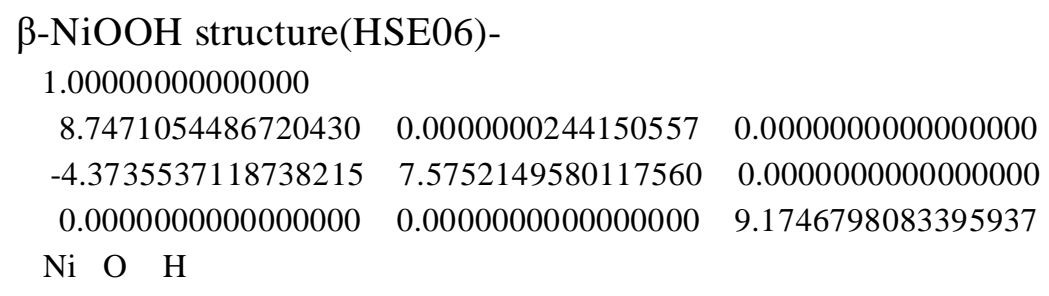
$\begin{array}{lll}18 & 36 & 18\end{array}$

Direct $\begin{array}{llll}0.0000000000000000 & 0.0000000000000000 & 0.4966660515229080\end{array}$ $\begin{array}{llll}0.0000000000000000 & 0.0000000000000000 & 0.9966660515229151\end{array}$ $\begin{array}{llll}0.0000000000000000 & 0.3333333429999996 & 0.4966660515229080\end{array}$ $\begin{array}{llll}0.0000000000000000 & 0.3333333429999996 & 0.9966660515229151\end{array}$ $\begin{array}{llll}0.0000000000000000 & 0.6666666870000029 & 0.4966660515229080\end{array}$ $\begin{array}{llll}0.0000000000000000 & 0.6666666870000029 & 0.9966660515229151\end{array}$ $0.33333334299999960 .0000000000000000 \quad 0.4966660515229080$ $\begin{array}{lllll}0.3333333429999996 & 0.0000000000000000 & 0.9966660515229151\end{array}$ $\begin{array}{lllll}0.3333333429999996 & 0.3333333429999996 & 0.4966660515229080\end{array}$ $\begin{array}{lllll}0.3333333429999996 & 0.3333333429999996 & 0.9966660515229151\end{array}$ $\begin{array}{lllll}0.3333333429999996 & 0.6666666870000029 & 0.4966660515229080\end{array}$ $\begin{array}{llll}0.3333333429999996 & 0.6666666870000029 & 0.9966660515229151\end{array}$ $\begin{array}{llll}0.6666666870000029 & 0.0000000000000000 & 0.4966660515229080\end{array}$ $\begin{array}{llll}0.6666666870000029 & 0.0000000000000000 & 0.9966660515229151\end{array}$ $\begin{array}{llll}0.6666666870000029 & 0.3333333429999996 & 0.4966660515229080\end{array}$ $\begin{array}{llll}0.6666666870000029 & 0.3333333429999996 & 0.9966660515229151\end{array}$ $\begin{array}{llll}0.6666666870000029 & 0.6666666870000029 & 0.4966660515229080\end{array}$ $\begin{array}{llll}0.6666666870000029 & 0.6666666870000029 & 0.9966660515229151\end{array}$ $\begin{array}{llll}0.1111111120000032 & 0.2222223280000009 & 0.1004277980151471\end{array}$ $\begin{array}{llll}0.1111111120000032 & 0.2222223280000009 & 0.6004278210151455\end{array}$ $\begin{array}{llll}0.1111111120000032 & 0.5555556420000016 & 0.1004277980151471\end{array}$ $\begin{array}{llll}0.1111111120000032 & 0.5555556420000016 & 0.6004278210151455\end{array}$ $\begin{array}{llll}0.1111111120000032 & 0.8888890149999966 & 0.1004277980151471\end{array}$ $\begin{array}{llll}0.1111111120000032 & 0.8888890149999966 & 0.6004278210151455\end{array}$ $\begin{array}{llll}0.4444444479999987 & 0.2222223280000009 & 0.1004277980151471\end{array}$ $\begin{array}{lllll}0.4444444479999987 & 0.2222223280000009 & 0.6004278210151455\end{array}$ $\begin{array}{lllll}0.4444444479999987 & 0.5555556420000016 & 0.1004277980151471\end{array}$ $0.4444444479999987 \quad 0.5555556420000016 \quad 0.6004278210151455$ $\begin{array}{lllll}0.4444444479999987 & 0.8888890149999966 & 0.1004277980151471\end{array}$ $\begin{array}{lllll}0.4444444479999987 & 0.8888890149999966 & 0.6004278210151455\end{array}$ $\begin{array}{llll}0.7777777909999983 & 0.2222223280000009 & 0.1004277980151471\end{array}$ $\begin{array}{llll}0.7777777909999983 & 0.2222223280000009 & 0.6004278210151455\end{array}$ 
$\begin{array}{llll}0.7777777909999983 & 0.5555556420000016 & 0.1004277980151471\end{array}$ $\begin{array}{lllll}0.7777777909999983 & 0.5555556420000016 & 0.6004278210151455\end{array}$ $\begin{array}{lllll}0.7777777909999983 & 0.8888890149999966 & 0.1004277980151471\end{array}$ $\begin{array}{lllll}0.7777777909999983 & 0.8888890149999966 & 0.6004278210151455\end{array}$ $\begin{array}{lllll}0.2222223280000009 & 0.1111111120000032 & 0.3869913864220109\end{array}$ $\begin{array}{lllll}0.2222223280000009 & 0.1111111120000032 & 0.8869913574220121\end{array}$ $\begin{array}{lllll}0.2222223280000009 & 0.4444444479999987 & 0.3869913864220109\end{array}$ $\begin{array}{lllll}0.2222223280000009 & 0.4444444479999987 & 0.8869913574220121\end{array}$ $\begin{array}{lllll}0.2222223280000009 & 0.7777777909999983 & 0.3869913864220109\end{array}$ $\begin{array}{lllll}0.2222223280000009 & 0.7777777909999983 & 0.8869913574220121\end{array}$ $\begin{array}{llll}0.5555556420000016 & 0.1111111120000032 & 0.3869913864220109\end{array}$ $\begin{array}{lllll}0.5555556420000016 & 0.1111111120000032 & 0.8869913574220121\end{array}$ $\begin{array}{lllll}0.5555556420000016 & 0.4444444479999987 & 0.3869913864220109\end{array}$ $\begin{array}{lllll}0.5555556420000016 & 0.4444444479999987 & 0.8869913574220121\end{array}$ $\begin{array}{llll}0.5555556420000016 & 0.7777777909999983 & 0.3869913864220109\end{array}$ $\begin{array}{llll}0.5555556420000016 & 0.7777777909999983 & 0.8869913574220121\end{array}$ $\begin{array}{llll}0.8888890149999966 & 0.1111111120000032 & 0.3869913864220109\end{array}$ $\begin{array}{lllll}0.8888890149999966 & 0.1111111120000032 & 0.8869913574220121\end{array}$ $\begin{array}{lllll}0.8888890149999966 & 0.4444444479999987 & 0.3869913864220109\end{array}$ $\begin{array}{lllll}0.8888890149999966 & 0.4444444479999987 & 0.8869913574220121\end{array}$ $\begin{array}{lllll}0.8888890149999966 & 0.7777777909999983 & 0.3869913864220109\end{array}$ $\begin{array}{lllll}0.8888890149999966 & 0.7777777909999983 & 0.8869913574220121\end{array}$ $\begin{array}{lllll}0.2222223280000009 & 0.1111109929999969 & 0.2808998850399291\end{array}$ $\begin{array}{lllll}0.2222223280000009 & 0.1111109929999969 & 0.7808999150399316\end{array}$ $\begin{array}{lllll}0.2222223280000009 & 0.4444443289999995 & 0.2808998850399291\end{array}$ $\begin{array}{lllll}0.2222223280000009 & 0.4444443289999995 & 0.7808999150399316\end{array}$ $\begin{array}{llllll}0.2222223280000009 & 0.7777776719999991 & 0.2808998850399291\end{array}$ $\begin{array}{llllll}0.2222223280000009 & 0.7777776719999991 & 0.7808999150399316\end{array}$ $\begin{array}{lllll}0.5555556420000016 & 0.1111109929999969 & 0.2808998850399291\end{array}$ $\begin{array}{lllll}0.5555556420000016 & 0.1111109929999969 & 0.7808999150399316\end{array}$ $\begin{array}{lllll}0.5555556420000016 & 0.4444443289999995 & 0.2808998850399291\end{array}$ $\begin{array}{llll}0.5555556420000016 & 0.4444443289999995 & 0.7808999150399316\end{array}$ $\begin{array}{lllll}0.5555556420000016 & 0.7777776719999991 & 0.2808998850399291\end{array}$ $\begin{array}{lllll}0.5555556420000016 & 0.7777776719999991 & 0.7808999150399316\end{array}$ $\begin{array}{lllll}0.8888890149999966 & 0.1111109929999969 & 0.2808998850399291\end{array}$ $\begin{array}{lllll}0.8888890149999966 & 0.1111109929999969 & 0.7808999150399316\end{array}$ $\begin{array}{lllll}0.8888890149999966 & 0.4444443289999995 & 0.2808998850399291\end{array}$ $\begin{array}{lllll}0.8888890149999966 & 0.4444443289999995 & 0.7808999150399316\end{array}$ $\begin{array}{lllll}0.8888890149999966 & 0.7777776719999991 & 0.2808998850399291\end{array}$ $\begin{array}{lllll}0.8888890149999966 & 0.7777776719999991 & 0.7808999150399316\end{array}$

$\beta$-NiOOH (with Ni vacancy) structure(HSE06)1.00000000000000 $\begin{array}{llll}8.7471054486720430 & 0.0000000244150557 & 0.0000000000000000\end{array}$ $\begin{array}{llll}-4.3735537118738215 & 7.5752149580117560 & 0.0000000000000000\end{array}$ $\begin{array}{llll}0.0000000000000000 & 0.0000000000000000 & 9.1746798083395937\end{array}$ $\mathrm{Ni} \mathrm{O} \quad \mathrm{H}$ 
$\begin{array}{lll}17 & 36 & 18\end{array}$

Direct

$\begin{array}{llll}0.0014866773982334 & 0.9993461737301459 & 0.4949079944531434\end{array}$ $\begin{array}{llll}0.9997514446269662 & 0.9994099008604138 & 0.9940725639366903\end{array}$ $\begin{array}{lllll}0.0000000000000000 & 0.3333333429999996 & 0.5014253040998398\end{array}$ $\begin{array}{llll}0.0000000000000000 & 0.3333333429999996 & 0.9997620995386072\end{array}$ $\begin{array}{llll}0.0014866773982334 & 0.6688071906680904 & 0.4949079944531434\end{array}$ $\begin{array}{llll}0.9997514446269662 & 0.6670082307665623 & 0.9940725639366903\end{array}$ $\begin{array}{lllll}0.3333333429999996 & 0.0000000000000000 & 0.0075041595249772\end{array}$ $\begin{array}{llll}0.3339871692698537 & 0.3318466656017662 & 0.4949079944531434\end{array}$ $\begin{array}{llll}0.3339234421395929 & 0.3335818983730334 & 0.9940725639366903\end{array}$ $\begin{array}{llll}0.3339871692698537 & 0.6688071906680904 & 0.4949079944531434\end{array}$ $\begin{array}{llll}0.3339234421395929 & 0.6670082307665623 & 0.9940725639366903\end{array}$ $\begin{array}{llll}0.6645261833319154 & 0.9993461737301459 & 0.4949079944531434\end{array}$ $\begin{array}{llll}0.6663251432334434 & 0.9994099008604138 & 0.9940725639366903\end{array}$ $\begin{array}{lllll}0.6645261833319154 & 0.3318466656017662 & 0.4949079944531434\end{array}$ $\begin{array}{llll}0.6663251432334434 & 0.3335818983730334 & 0.9940725639366903\end{array}$ $\begin{array}{llll}0.6666666870000029 & 0.6666666870000029 & 0.4938474913426063\end{array}$ $\begin{array}{llll}0.6666666870000029 & 0.6666666870000029 & 0.9955085689828849\end{array}$ $\begin{array}{llll}0.1178869342619961 & 0.2154465057380008 & 0.1001434361992395\end{array}$ $\begin{array}{lllll}0.1185278997520953 & 0.2148055402479088 & 0.5998672386871320\end{array}$ $\begin{array}{lllll}0.1178869342619961 & 0.5691072865240017 & 0.1001434361992395\end{array}$ $\begin{array}{llll}0.1185278997520953 & 0.5703892175041858 & 0.5998672386871320\end{array}$ $\begin{array}{llll}0.0927301395067488 & 0.8796985287533730 & 0.1018408443627123\end{array}$ $\begin{array}{llll}0.0683458330725841 & 0.8675063755362942 & 0.6083499930778231\end{array}$ $\begin{array}{llll}0.4536349342466224 & 0.2406033004932553 & 0.1018408443627123\end{array}$ $\begin{array}{lllll}0.4658270874637012 & 0.2649876069274200 & 0.6083499930778231\end{array}$ $\begin{array}{llll}0.4315321354688280 & 0.5490994857344234 & 0.0975096684334815\end{array}$ $\begin{array}{llll}0.4297146239014324 & 0.5481907299507256 & 0.5937984430026759\end{array}$ $\begin{array}{llll}0.4536349342466224 & 0.8796985287533730 & 0.1018408443627123\end{array}$ $\begin{array}{llll}0.4658270874637012 & 0.8675063755362942 & 0.6083499930778231\end{array}$ $\begin{array}{llll}0.7642261464759983 & 0.2154465057380008 & 0.1001434361992395\end{array}$ $\begin{array}{lllll}0.7629442154958141 & 0.2148055402479088 & 0.5998672386871320\end{array}$ $\begin{array}{lllll}0.7842339472655766 & 0.5490994857344234 & 0.0975096684334815\end{array}$ $\begin{array}{llll}0.7851427030492744 & 0.5481907299507256 & 0.5937984430026759\end{array}$ $\begin{array}{lllll}0.7842339472655766 & 0.9018013275311674 & 0.0975096684334815\end{array}$ $\begin{array}{llll}0.7851427030492744 & 0.9036188390985629 & 0.5937984430026759\end{array}$ $\begin{array}{lllll}0.2186949518151806 & 0.1146384881848235 & 0.3934142679612123\end{array}$ $\begin{array}{lllll}0.2207157194105278 & 0.1126177205894834 & 0.8877796813494712\end{array}$ $\begin{array}{llll}0.2279859830779358 & 0.4473262755389698 & 0.3867550964861266\end{array}$ $\begin{array}{lllll}0.2278098267694091 & 0.4472381973847064 & 0.8853265312605885\end{array}$ $\begin{array}{llll}0.2186949518151806 & 0.7707230386303578 & 0.3934142679612123\end{array}$ $\begin{array}{llll}0.2207157194105278 & 0.7747645738210664 & 0.8877796813494712\end{array}$ $\begin{array}{llll}0.5626103943696421 & 0.1146384881848235 & 0.3934142679612123\end{array}$ $\begin{array}{lllll}0.5585688591789335 & 0.1126177205894834 & 0.8877796813494712\end{array}$ $\begin{array}{llll}0.5494465145867906 & 0.4322261931735483 & 0.3834143122412854\end{array}$ $\begin{array}{llll}0.5516807722327783 & 0.4366947084655308 & 0.8832332441685651\end{array}$ $\begin{array}{lllll}0.5494465145867906 & 0.7838869184132022 & 0.3834143122412854\end{array}$ $\begin{array}{llll}0.5516807722327783 & 0.7816526607672216 & 0.8832332441685651\end{array}$ 
$\begin{array}{llll}0.8860071874610256 & 0.1053474569220683 & 0.3867550964861266\end{array}$ $\begin{array}{llll}0.8860952656152890 & 0.1055236132305879 & 0.8853265312605885\end{array}$ $\begin{array}{lllll}0.8860071874610256 & 0.4473262755389698 & 0.3867550964861266\end{array}$ $\begin{array}{lllll}0.8860952656152890 & 0.4472381973847064 & 0.8853265312605885\end{array}$ $\begin{array}{llll}0.9011072698264471 & 0.7838869184132022 & 0.3834143122412854\end{array}$ $\begin{array}{llll}0.8966387545344645 & 0.7816526607672216 & 0.8832332441685651\end{array}$ $\begin{array}{llll}0.1983335297637865 & 0.1349997912362113 & 0.2922442179655249\end{array}$ $\begin{array}{lllll}0.2169204785402741 & 0.1164128424597237 & 0.7817996194644863\end{array}$ $\begin{array}{llll}0.2434016267987502 & 0.4550339783993778 & 0.2812594575228360\end{array}$ $\begin{array}{llll}0.2414987472668102 & 0.4540825386334149 & 0.7798159092499475\end{array}$ $\begin{array}{lllll}0.1983335297637865 & 0.7300000755275704 & 0.2922442179655249\end{array}$ $\begin{array}{lllll}0.2169204785402741 & 0.7671739730805314 & 0.7817996194644863\end{array}$ $\begin{array}{llll}0.6033332384724304 & 0.1349997912362113 & 0.2922442179655249\end{array}$ $\begin{array}{lllll}0.5661593409194694 & 0.1164128424597237 & 0.7817996194644863\end{array}$ $\begin{array}{llll}0.5337917168407245 & 0.4009164786814736 & 0.2795467185084206\end{array}$ $\begin{array}{llll}0.5401718244322069 & 0.4136766938644101 & 0.7774961881413986\end{array}$ $\begin{array}{llll}0.5337917168407245 & 0.7995415971592763 & 0.2795467185084206\end{array}$ $\begin{array}{llll}0.5401718244322069 & 0.7931614895677939 & 0.7774961881413986\end{array}$ $\begin{array}{lllll}0.8782993656006184 & 0.0899316942012476 & 0.2812594575228360\end{array}$ $\begin{array}{lllll}0.8792508053665884 & 0.0918345737331876 & 0.7798159092499475\end{array}$ $\begin{array}{lllll}0.8782993656006184 & 0.4550339783993778 & 0.2812594575228360\end{array}$ $\begin{array}{llll}0.8792508053665884 & 0.4540825386334149 & 0.7798159092499475\end{array}$ $\begin{array}{llll}0.9324168653185154 & 0.7995415971592763 & 0.2795467185084206\end{array}$ $\begin{array}{llll}0.9196566501355861 & 0.7931614895677939 & 0.7774961881413986\end{array}$

$\beta-\mathrm{NiOOH}$ (with O vacancy) structure(HSE06)-

1.00000000000000

$\begin{array}{llll}8.7471054486720430 & 0.0000000244150557 & 0.0000000000000000\end{array}$

$\begin{array}{llll}-4.3735537118738215 & 7.5752149580117560 & 0.0000000000000000\end{array}$

$\begin{array}{llll}0.0000000000000000 & 0.0000000000000000 & 9.1746798083395937\end{array}$

$\mathrm{Ni} \quad \mathrm{O} \quad \mathrm{H}$

$\begin{array}{lll}18 & 35 & 18\end{array}$

Direct

$\begin{array}{llll}0.0093400005658779 & 0.0046700002829283 & 0.4905308752684476\end{array}$ $\begin{array}{llll}0.0007378377512737 & 0.0003689188756297 & 0.9940801342950181\end{array}$ $\begin{array}{lllll}0.9980544164155702 & 0.3294421758311117 & 0.5052104785388707\end{array}$ $\begin{array}{llll}0.0000316176273429 & 0.3333965782546926 & 0.9989957626308481\end{array}$ $\begin{array}{lllll}0.9980544164155702 & 0.6686122705844326 & 0.5052104785388707\end{array}$ $\begin{array}{lllll}0.0000316176273429 & 0.6666350693726599 & 0.9989957626308481\end{array}$ $\begin{array}{llll}0.3344330396331614 & 0.0021993932663236 & 0.4947924958987215\end{array}$ $\begin{array}{llll}0.3333011413584046 & 0.9999355967168242 & 0.9938177502664658\end{array}$ $\begin{array}{lllll}0.3344330396331614 & 0.3322336463668378 & 0.4947924958987215\end{array}$ $\begin{array}{lllll}0.3333011413584046 & 0.3333655446415946 & 0.9938177502664658\end{array}$ $\begin{array}{lllll}0.3372245101688875 & 0.6686122705844326 & 0.5052104785388707\end{array}$ $\begin{array}{llll}0.3332701077453066 & 0.6666350693726599 & 0.9989957626308481\end{array}$ $\begin{array}{lllll}0.6619966867170604 & 0.0046700002829283 & 0.4905308752684476\end{array}$ $\begin{array}{lllll}0.6662977681243731 & 0.0003689188756297 & 0.9940801342950181\end{array}$ $\begin{array}{llll}0.6644672937336793 & 0.3322336463668378 & 0.4947924958987215\end{array}$ $\begin{array}{llll}0.6667310902831858 & 0.3333655446415946 & 0.9938177502664658\end{array}$ 
$\begin{array}{llll}0.6619966867170604 & 0.6573266864341250 & 0.4905308752684476\end{array}$ $\begin{array}{lllll}0.6662977681243731 & 0.6659288492487292 & 0.9940801342950181\end{array}$ $\begin{array}{lllll}0.1105715592040681 & 0.2214949279742058 & 0.0990940274650356\end{array}$ $\begin{array}{lllll}0.1013808279761719 & 0.2039783292012345 & 0.6027430761697232\end{array}$ $\begin{array}{llll}0.1111111120000032 & 0.5555556420000016 & 0.1032898405187623\end{array}$ $\begin{array}{lllll}0.1111111120000032 & 0.5555556420000016 & 0.6153691906576171\end{array}$ $\begin{array}{lllll}0.1105715592040681 & 0.8890768622298637 & 0.0990940274650356\end{array}$ $\begin{array}{lllll}0.1013808279761719 & 0.8974027297749245 & 0.6027430761697232\end{array}$ $\begin{array}{lllll}0.4444444479999987 & 0.2222223280000009 & 0.0953964561885243\end{array}$ $\begin{array}{llllll}0.4444444479999987 & 0.2222223280000009 & 0.5939705777354831\end{array}$ $\begin{array}{lllll}0.4451718480257938 & 0.5560951947959367 & 0.0990940274650356\end{array}$ $\begin{array}{lllll}0.4626884467987651 & 0.5652859260238259 & 0.6027430761697232\end{array}$ $\begin{array}{lllll}0.4451718480257938 & 0.8890768622298637 & 0.0990940274650356\end{array}$ $\begin{array}{lllll}0.4626884467987651 & 0.8974027297749245 & 0.6027430761697232\end{array}$ $\begin{array}{llll}0.7775899437701312 & 0.2214949279742058 & 0.0990940274650356\end{array}$ $\begin{array}{lllll}0.7692640762250775 & 0.2039783292012345 & 0.6027430761697232\end{array}$ $\begin{array}{lllll}0.7775899437701312 & 0.5560951947959367 & 0.0990940274650356\end{array}$ $\begin{array}{lllll}0.7692640762250775 & 0.5652859260238259 & 0.6027430761697232\end{array}$ $\begin{array}{lllll}0.7777777909999983 & 0.8888890149999966 & 0.0987266657458790\end{array}$ $\begin{array}{lllll}0.2226810836036677 & 0.1113404898018260 & 0.3750510176349025\end{array}$ $\begin{array}{lllll}0.2212090979810810 & 0.1106044969905469 & 0.8839028619795855\end{array}$ $\begin{array}{lllll}0.2211537115974735 & 0.4455130644025189 & 0.3955915421591456\end{array}$ $\begin{array}{lllll}0.2207885538649208 & 0.4458782221350859 & 0.8878397948706152\end{array}$ $\begin{array}{lllll}0.2211537115974735 & 0.7756405581949437 & 0.3955915421591456\end{array}$ $\begin{array}{lllll}0.2207885538649208 & 0.7749102427298169 & 0.8878397948706152\end{array}$ $\begin{array}{lllll}0.5553262641981718 & 0.1113404898018260 & 0.3750510176349025\end{array}$ $\begin{array}{lllll}0.5560622570094580 & 0.1106044969905469 & 0.8839028619795855\end{array}$ $\begin{array}{lllll}0.5553262641981718 & 0.4439856923963319 & 0.3750510176349025\end{array}$ $\begin{array}{lllll}0.5560622570094580 & 0.4454576780189186 & 0.8839028619795855\end{array}$ $\begin{array}{lllll}0.5680573897234993 & 0.7840286648617649 & 0.3926687347648468\end{array}$ $\begin{array}{lllll}0.5529455290305023 & 0.7764727345152380 & 0.8850502251152292\end{array}$ $\begin{array}{llll}0.8826381411382300 & 0.0986093642765056 & 0.3926687347648468\end{array}$ $\begin{array}{llll}0.8901940714847569 & 0.1137212249695025 & 0.8850502251152292\end{array}$ $\begin{array}{lllll}0.8910262478050512 & 0.4455130644025189 & 0.3955915421591456\end{array}$ $\begin{array}{lllll}0.8917565632701780 & 0.4458782221350859 & 0.8878397948706152\end{array}$ $\begin{array}{lllll}0.8826381411382300 & 0.7840286648617649 & 0.3926687347648468\end{array}$ $\begin{array}{lllll}0.8901940714847569 & 0.7764727345152380 & 0.8850502251152292\end{array}$ $\begin{array}{lllll}0.2205402849389344 & 0.1102699714694637 & 0.2694473519002401\end{array}$ $\begin{array}{lllll}0.2158423507465699 & 0.1079210043732743 & 0.7777552763657170\end{array}$ $\begin{array}{llllll}0.2195547207338819 & 0.4471119362661184 & 0.2897267577637663\end{array}$ $\begin{array}{lllll}0.2155513711885746 & 0.4511152858114258 & 0.7821943044228306\end{array}$ $\begin{array}{llllll}0.2195547207338819 & 0.7724424574677471 & 0.2897267577637663\end{array}$ $\begin{array}{lllll}0.2155513711885746 & 0.7644357583771395 & 0.7821943044228306\end{array}$ $\begin{array}{lllll}0.5563966635305349 & 0.1102699714694637 & 0.2694473519002401\end{array}$ $\begin{array}{lllll}0.5587456306267171 & 0.1079210043732743 & 0.7777552763657170\end{array}$ $\begin{array}{lllll}0.5563966635305349 & 0.4461263720610660 & 0.2694473519002401\end{array}$ $\begin{array}{llllll}0.5587456306267171 & 0.4508243062534234 & 0.7777552763657170\end{array}$ $\begin{array}{lllll}0.5761031201267812 & 0.7880514110633854 & 0.2865857065905644\end{array}$ $\begin{array}{llll}0.5350241502112141 & 0.7675119261056054 & 0.7800219144959186\end{array}$ 
$\begin{array}{llll}0.8786152759366104 & 0.0905635148732173 & 0.2865857065905644\end{array}$ $\begin{array}{lllll}0.8991547608943904 & 0.1316424847887774 & 0.7800219144959186\end{array}$ $\begin{array}{lllll}0.8942242295322416 & 0.4471119362661184 & 0.2897267577637663\end{array}$ $\begin{array}{lllll}0.9022309286228491 & 0.4511152858114258 & 0.7821943044228306\end{array}$ $\begin{array}{lllll}0.8786152759366104 & 0.7880514110633854 & 0.2865857065905644\end{array}$ $\begin{array}{lllll}0.8991547608943904 & 0.7675119261056054 & 0.7800219144959186\end{array}$

$\beta-\mathrm{NiOOH}$ (with $\mathrm{H}$ vacancy) structure(HSE06)-

1.00000000000000

$\begin{array}{llll}8.7471054486720430 & 0.0000000244150557 & 0.0000000000000000\end{array}$

$\begin{array}{llll}-4.3735537118738215 & 7.5752149580117560 & 0.0000000000000000\end{array}$

$\begin{array}{llll}0.0000000000000000 & 0.0000000000000000 & 9.1746798083395937\end{array}$

$\mathrm{Ni} \quad \mathrm{O} \quad \mathrm{H}$

$\begin{array}{lll}18 & 36 & 17\end{array}$

Direct

$\begin{array}{llll}0.0012044101190867 & 0.9987955898809133 & 0.4983302461286527\end{array}$

$\begin{array}{llll}0.9975326284045849 & 0.0024673715954151 & 0.9931192127537329\end{array}$

$\begin{array}{lllll}0.0012044101190867 & 0.3357421632381943 & 0.4983302461286527\end{array}$

$\begin{array}{lllll}0.9975326284045849 & 0.3283985998091765 & 0.9931192127537329\end{array}$

$\begin{array}{lllll}0.0023092456879610 & 0.6678213098439869 & 0.4882685714120356\end{array}$

$\begin{array}{lllll}0.0073566044772875 & 0.6703449892386573 & 0.9921293631599113\end{array}$

$\begin{array}{lllll}0.3321787201560156 & 0.9976907543120390 & 0.4882685714120356\end{array}$

$\begin{array}{lllll}0.3296550407613523 & 0.9926433955227125 & 0.9921293631599113\end{array}$

$\begin{array}{lllll}0.3450982016797965 & 0.3392157723398981 & 0.4965163139483053\end{array}$

$\begin{array}{lllll}0.3318795502660237 & 0.3326064466330081 & 0.0125784315220656\end{array}$

$\begin{array}{lllll}0.3321787201560156 & 0.6678213098439869 & 0.4882685714120356\end{array}$

$\begin{array}{lllll}0.3296550407613523 & 0.6703449892386573 & 0.9921293631599113\end{array}$

$\begin{array}{lllll}0.6642578667618153 & 0.9987955898809133 & 0.4983302461286527\end{array}$

$\begin{array}{llll}0.6716014301908331 & 0.0024673715954151 & 0.9931192127537329\end{array}$

$\begin{array}{lllll}0.6607842576600973 & 0.3392157723398981 & 0.4965163139483053\end{array}$

$\begin{array}{lllll}0.6673935833669944 & 0.3326064466330081 & 0.0125784315220656\end{array}$

$\begin{array}{llllll}0.6607842576600973 & 0.6549018283202059 & 0.4965163139483053\end{array}$

$\begin{array}{lllll}0.6673935833669944 & 0.6681204797339788 & 0.0125784315220656\end{array}$

$\begin{array}{llll}0.0919595020505355 & 0.2126465230252776 & 0.0965659408847159\end{array}$

$\begin{array}{lllll}0.1364161387939617 & 0.2348748413969801 & 0.6013057194607896\end{array}$

$\begin{array}{lllll}0.1210142104252725 & 0.5753618388505330 & 0.0932930748211831\end{array}$

$\begin{array}{lllll}0.1187311895864553 & 0.5707957971728987 & 0.5899412135746473\end{array}$

$\begin{array}{lllll}0.1210142104252725 & 0.8789859165747345 & 0.0932930748211831\end{array}$

$\begin{array}{lllll}0.1187311895864553 & 0.8812689374135445 & 0.5899412135746473\end{array}$

$\begin{array}{lllll}0.4247955971976296 & 0.1829246263952413 & 0.1141901622170423\end{array}$

$\begin{array}{lllll}0.4535348016867147 & 0.2404030353734399 & 0.6002817917584125\end{array}$

$\begin{array}{lllll}0.4247955971976296 & 0.5752044928023707 & 0.1141901622170423\end{array}$

$\begin{array}{lllll}0.4535348016867147 & 0.5464652883132786 & 0.6002817917584125\end{array}$

$\begin{array}{lllll}0.4246382511494673 & 0.8789859165747345 & 0.0932930748211831\end{array}$

$\begin{array}{lllll}0.4292042928270945 & 0.8812689374135445 & 0.5899412135746473\end{array}$

$\begin{array}{lllll}0.7873535959747215 & 0.2126465230252776 & 0.0965659408847159\end{array}$

$\begin{array}{lllll}0.7651252776030120 & 0.2348748413969801 & 0.6013057194607896\end{array}$

$\begin{array}{llll}0.8170754926047579 & 0.5752044928023707 & 0.1141901622170423\end{array}$ 
$\begin{array}{llll}0.7595970836265593 & 0.5464652883132786 & 0.6002817917584125\end{array}$ $\begin{array}{lllll}0.7873535959747215 & 0.9080406249494644 & 0.0965659408847159\end{array}$ $\begin{array}{lllll}0.7651252776030120 & 0.8635839882060381 & 0.6013057194607896\end{array}$ $\begin{array}{lllll}0.2395643248032826 & 0.1246071776508728 & 0.3870483298289216\end{array}$ $\begin{array}{llll}0.2227642755580135 & 0.1041814994897976 & 0.8911892607069944\end{array}$ $\begin{array}{lllll}0.2395643248032826 & 0.4482903791524038 & 0.3870483298289216\end{array}$ $\begin{array}{lllll}0.2227642755580135 & 0.4519160080682099 & 0.8911892607069944\end{array}$ $\begin{array}{lllll}0.2222223280000009 & 0.7777777909999983 & 0.3661011796963436\end{array}$ $\begin{array}{llll}0.2222223280000009 & 0.7777777909999983 & 0.8780070927871009\end{array}$ $\begin{array}{lllll}0.5517097108475824 & 0.1246071776508728 & 0.3870483298289216\end{array}$ $\begin{array}{lllll}0.5480840819317905 & 0.1041814994897976 & 0.8911892607069944\end{array}$ $\begin{array}{llllll}0.5555556420000016 & 0.4444444479999987 & 0.3915907992207579\end{array}$ $\begin{array}{lllll}0.5555556420000016 & 0.4444444479999987 & 0.9046402725520082\end{array}$ $\begin{array}{lllll}0.5517097108475824 & 0.7604357941967237 & 0.3870483298289216\end{array}$ $\begin{array}{lllll}0.5480840819317905 & 0.7772358434419857 & 0.8911892607069944\end{array}$ $\begin{array}{lllll}0.8888890149999966 & 0.1111111120000032 & 0.3938126616393447\end{array}$ $\begin{array}{lllll}0.8888890149999966 & 0.1111111120000032 & 0.8683242907238409\end{array}$ $\begin{array}{lllll}0.8753929493491270 & 0.4482903791524038 & 0.3870483298289216\end{array}$ $\begin{array}{lllll}0.8958186275102022 & 0.4519160080682099 & 0.8911892607069944\end{array}$ $\begin{array}{lllll}0.8753929493491270 & 0.7604357941967237 & 0.3870483298289216\end{array}$ $\begin{array}{lllll}0.8958186275102022 & 0.7772358434419857 & 0.8911892607069944\end{array}$ $\begin{array}{lllll}0.2704621364875663 & 0.1377428615764416 & 0.2828579298496336\end{array}$ $\begin{array}{lllll}0.2240155834550848 & 0.1093538267417458 & 0.7851853955871277\end{array}$ $\begin{array}{lllll}0.2704621364875663 & 0.4660522689111417 & 0.2828579298496336\end{array}$ $\begin{array}{lllll}0.2240155834550848 & 0.4479947507133346 & 0.7851853955871277\end{array}$ $\begin{array}{lllll}0.2222223280000009 & 0.7777776719999991 & 0.2603301429818643\end{array}$ $\begin{array}{lllll}0.2222223280000009 & 0.7777776719999991 & 0.7710326341385993\end{array}$ $\begin{array}{lllll}0.5339477020888594 & 0.1377428615764416 & 0.2828579298496336\end{array}$ $\begin{array}{lllll}0.5520052202866665 & 0.1093538267417458 & 0.7851853955871277\end{array}$ $\begin{array}{lllll}0.5555556420000016 & 0.4444443289999995 & 0.7990230190078620\end{array}$ $\begin{array}{lllll}0.5339477020888594 & 0.7295378635124266 & 0.2828579298496336\end{array}$ $\begin{array}{llll}0.5520052202866665 & 0.7759844165449223 & 0.7851853955871277\end{array}$ $\begin{array}{llll}0.8888890149999966 & 0.1111109929999969 & 0.2872477363480783\end{array}$ $\begin{array}{lllll}0.8888890149999966 & 0.1111109929999969 & 0.7631766353381408\end{array}$ $\begin{array}{lllll}0.8622571464235520 & 0.4660522689111417 & 0.2828579298496336\end{array}$ $\begin{array}{lllll}0.8906461812582478 & 0.4479947507133346 & 0.7851853955871277\end{array}$ $\begin{array}{lllll}0.8622571464235520 & 0.7295378635124266 & 0.2828579298496336\end{array}$ $\begin{array}{llll}0.8906461812582478 & 0.7759844165449223 & 0.7851853955871277\end{array}$

$\beta-\mathrm{NiOOH}$ (with OH vacancy) structure(HSE06)1.00000000000000

$\begin{array}{rrr}8.7471054486720430 & 0.0000000244150557 & 0.0000000000000000 \\ -4.3735537118738215 & 7.5752149580117560 & 0.0000000000000000 \\ 0.0000000000000000 & 0.0000000000000000 & 9.1746798083395937\end{array}$

$\mathrm{Ni} \mathrm{O} \quad \mathrm{H}$

$\begin{array}{lll}18 & 35 & 17\end{array}$

Direct

$\begin{array}{llll}0.0001050145177430 & 0.0000525072588857 & 0.5031958477507175\end{array}$

$\begin{array}{llll}0.9931638785283425 & 0.9965819392641819 & 0.9971700484360539\end{array}$ 
$\begin{array}{llll}0.9962207387717044 & 0.3371126042282953 & 0.4964604588123862\end{array}$ $\begin{array}{llll}0.9999636909659060 & 0.3333696520340865 & 0.0043151643280552\end{array}$ $\begin{array}{llll}0.9962207387717044 & 0.6591081645434116 & 0.4964604588123862\end{array}$ $\begin{array}{lllll}0.9999636909659060 & 0.6665940689318575 & 0.0043151643280552\end{array}$ $\begin{array}{llll}0.3332808357411139 & 0.0000525072588857 & 0.5031958477507175\end{array}$ $\begin{array}{llll}0.3367514037358248 & 0.9965819392641819 & 0.9971700484360539\end{array}$ $\begin{array}{llll}0.3332808357411139 & 0.3332283284822566 & 0.5031958477507175\end{array}$ $\begin{array}{llll}0.3367514037358248 & 0.3401694644716571 & 0.9971700484360539\end{array}$ $\begin{array}{lllll}0.3368796301474646 & 0.6684398305737318 & 0.4929899377107532\end{array}$ $\begin{array}{llll}0.3388568880538827 & 0.6694284595269693 & 0.9913203903532448\end{array}$ $\begin{array}{llll}0.6648935434262739 & 0.9964537128525421 & 0.4929899377107532\end{array}$ $\begin{array}{llll}0.6639049144730365 & 0.9944764549461098 & 0.9913203903532448\end{array}$ $\begin{array}{llll}0.6742252094565941 & 0.3371126042282953 & 0.4964604588123862\end{array}$ $\begin{array}{llll}0.6667393050681483 & 0.3333696520340865 & 0.0043151643280552\end{array}$ $\begin{array}{lllll}0.6648935434262739 & 0.6684398305737318 & 0.4929899377107532\end{array}$ $\begin{array}{lllll}0.6639049144730365 & 0.6694284595269693 & 0.9913203903532448\end{array}$ $\begin{array}{lllll}0.1198445143782934 & 0.2134889256217107 & 0.0875923080342886\end{array}$ $\begin{array}{lllll}0.0952658556612533 & 0.2380675843387507 & 0.6077894595851845\end{array}$ $\begin{array}{lllll}0.1297497863305779 & 0.5648749791652818 & 0.1119110675461101\end{array}$ $\begin{array}{lllll}0.0932006257253803 & 0.5466003988626866 & 0.5995351797533530\end{array}$ $\begin{array}{llll}0.1198445143782934 & 0.9063558197565840 & 0.0875923080342886\end{array}$ $\begin{array}{lllll}0.0952658556612533 & 0.8571985023225110 & 0.6077894595851845\end{array}$ $\begin{array}{lllll}0.4269776432434114 & 0.2134889256217107 & 0.0875923080342886\end{array}$ $\begin{array}{lllll}0.4761349606774843 & 0.2380675843387507 & 0.6077894595851845\end{array}$ $\begin{array}{llll}0.4539982341784352 & 0.5746632143568746 & 0.0931603267389178\end{array}$ $\begin{array}{llll}0.4525799332081633 & 0.5718266124163165 & 0.5946873085585977\end{array}$ $\begin{array}{lllll}0.4539982341784352 & 0.8793352288215601 & 0.0931603267389178\end{array}$ $\begin{array}{lllll}0.4525799332081633 & 0.8807535297918392 & 0.5946873085585977\end{array}$ $\begin{array}{llll}0.7684584538347181 & 0.2035836536694262 & 0.1119110675461101\end{array}$ $\begin{array}{lllll}0.7867330341373062 & 0.2401328142746237 & 0.5995351797533530\end{array}$ $\begin{array}{llll}0.7684584538347181 & 0.5648749791652818 & 0.1119110675461101\end{array}$ $\begin{array}{llll}0.7867330341373062 & 0.5466003988626866 & 0.5995351797533530\end{array}$ $\begin{array}{lllll}0.7586702186431253 & 0.8793352288215601 & 0.0931603267389178\end{array}$ $\begin{array}{lllll}0.7615068205836764 & 0.8807535297918392 & 0.5946873085585977\end{array}$ $\begin{array}{llll}0.2222223280000009 & 0.1111111120000032 & 0.3926867649400876\end{array}$ $\begin{array}{lllll}0.2103877275415371 & 0.4464525985213683 & 0.3892305012540831\end{array}$ $\begin{array}{llll}0.2285681169211387 & 0.4513233909384340 & 0.8878725951457440\end{array}$ $\begin{array}{lllll}0.2103877275415371 & 0.7639350400201579 & 0.3892305012540831\end{array}$ $\begin{array}{llll}0.2285681169211387 & 0.7772446369827080 & 0.8878725951457440\end{array}$ $\begin{array}{llll}0.5693983929798350 & 0.1229457124584670 & 0.3892305012540831\end{array}$ $\begin{array}{lllll}0.5560887960172920 & 0.1047653230788654 & 0.8878725951457440\end{array}$ $\begin{array}{llll}0.5693983929798350 & 0.4464525985213683 & 0.3892305012540831\end{array}$ $\begin{array}{lllll}0.5560887960172920 & 0.4513233909384340 & 0.8878725951457440\end{array}$ $\begin{array}{lllll}0.5555556420000016 & 0.7777777909999983 & 0.3695525276841138\end{array}$ $\begin{array}{llll}0.5555556420000016 & 0.7777777909999983 & 0.8739233206161074\end{array}$ $\begin{array}{llll}0.8868808644786270 & 0.1229457124584670 & 0.3892305012540831\end{array}$ $\begin{array}{lllll}0.8820100720615685 & 0.1047653230788654 & 0.8878725951457440\end{array}$ $\begin{array}{lllll}0.8888890149999966 & 0.4444444479999987 & 0.3844915302366019\end{array}$ $\begin{array}{llll}0.8888890149999966 & 0.4444444479999987 & 0.8957410285124112\end{array}$ 


\begin{abstract}
$\begin{array}{llll}0.8868808644786270 & 0.7639350400201579 & 0.3892305012540831\end{array}$
$\begin{array}{llll}0.8820100720615685 & 0.7772446369827080 & 0.8878725951457440\end{array}$

$\begin{array}{llll}0.2222223280000009 & 0.1111109929999969 & 0.2871202962723842\end{array}$

$\begin{array}{llll}0.1973220976513872 & 0.4598166209373389 & 0.2848013609200706\end{array}$

$\begin{array}{llll}0.2303108995323342 & 0.4509215213061566 & 0.7816441963996041\end{array}$

$\begin{array}{llll}0.1973220976513872 & 0.7375051497140603 & 0.2848013609200706\end{array}$

$\begin{array}{llll}0.2303108995323342 & 0.7793890512261470 & 0.7816441963996041\end{array}$

$\begin{array}{llll}0.5958281642859404 & 0.1360112233486106 & 0.2848013609200706\end{array}$

$\begin{array}{llll}0.5539442627738538 & 0.1030224214676636 & 0.7816441963996041\end{array}$

$\begin{array}{lllll}0.5958281642859404 & 0.4598166209373389 & 0.2848013609200706\end{array}$

$\begin{array}{llll}0.5539442627738538 & 0.4509215213061566 & 0.7816441963996041\end{array}$

$\begin{array}{llll}0.5555556420000016 & 0.7777776719999991 & 0.2639615860994695\end{array}$

$\begin{array}{llll}0.5555556420000016 & 0.7777776719999991 & 0.7668557179840434\end{array}$

$\begin{array}{llll}0.8735167230626573 & 0.1360112233486106 & 0.2848013609200706\end{array}$

$\begin{array}{llll}0.8824118226938396 & 0.1030224214676636 & 0.7816441963996041\end{array}$

$\begin{array}{llll}0.8888890149999966 & 0.4444443289999995 & 0.2781289065302701\end{array}$

$\begin{array}{llll}0.8888890149999966 & 0.4444443289999995 & 0.7897960573684344\end{array}$

$\begin{array}{llll}0.8735167230626573 & 0.7375051497140603 & 0.2848013609200706\end{array}$

$\begin{array}{llll}0.8824118226938396 & 0.7793890512261470 & 0.7816441963996041\end{array}$
\end{abstract}

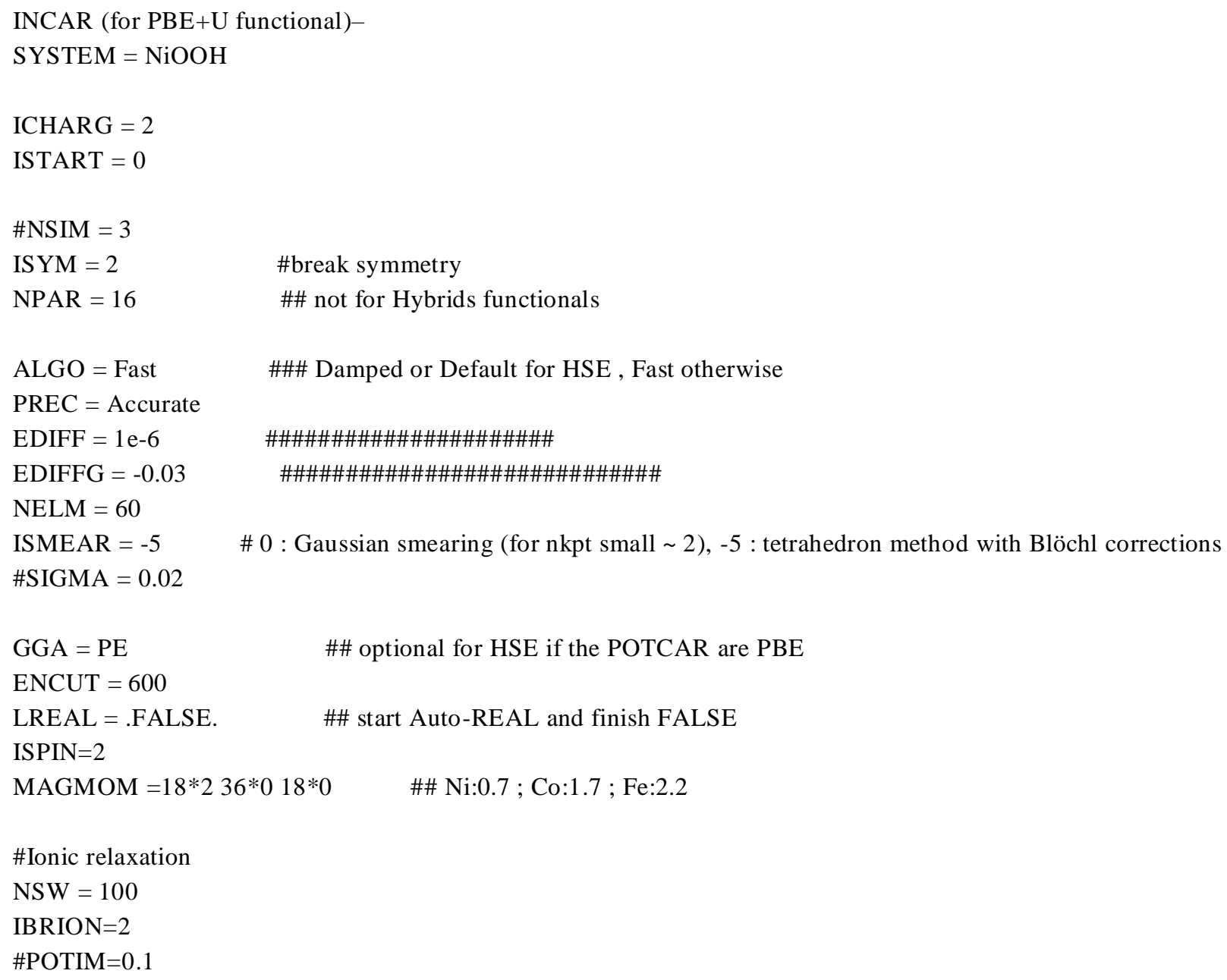


ISIF=3 \#relax the cell parameters

\#DFT+U

LDAU = .TRUE. \# turn on DFT $+\mathrm{U}$

LDAUTYPE $=2 \quad$ \# various formalism, keep 2

LDAUL $=2-1-1 \quad \#-1$ do not activate DFT+U, 2 activate DFT+U

$\mathrm{LDAUU}=5.500 \quad$ \# U value for each kind of atom (order of the POSCAR)

LDAUJ $=\begin{array}{llll}0 & 0 & 0\end{array} \quad$ \# dont change

LDAUPRINT $=1$ \# ??? 